\title{
Time-Varying Maximum Transition Run Constraints
}

\author{
T. Lei Poo, Member, IEEE, and Brian H. Marcus, Fellow, IEEE
}

\begin{abstract}
Maximum transition run (MTR) constrained systems are used to improve detection performance in storage channels. Recently, there has been a growing interest in time-varying MTR (TMTR) systems, after such codes were observed to eliminate certain error events and thus provide high coding gain for $E^{n} \mathrm{PR} 4$ channels for $n=2,3$.

In this work, TMTR constraints parameterized by a vector, whose coordinates specify periodically the maximum runlengths of 1 's ending at the positions, are investigated. A canonical way to classify such constraints and simplify their minimal graph presentations is introduced. It is shown that there is a particularly simple presentation for a special class of TMTR constraints and explicit descriptions of their characteristic equations are derived. New upper bounds on the capacity of TMTR constraints are established, and an explicit linear ordering by capacity of all tight TMTR constraints up to period 4 is given. For MTR constrained systems with unconstrained positions, it is shown that the set of sequences restricted to the constrained positions yields a natural TMTR constraint. Using TMTR constraints, a new upper bound on the tradeoff function for MTR systems that relates the density of unconstrained positions to the maximum code rates is determined.
\end{abstract}

Index Terms-Capacity, constrained systems, maximum transition run (MTR), time varying, tradeoff function, upper bounds.

\section{INTRODUCTION}

$\mathbf{I}$ $\mathrm{T}$ is well known that maximum transition run (MTR) constrained codes [9] are a means of achieving coding gain for high-density magnetic recording. In the non-return to zero inverse (NRZI) domain, where a 0 corresponds to no transition and a 1 corresponds to a transition, the $\operatorname{MTR}(j)$ codes limit the run of 1 's to be at most $j$, i.e., at most $j$ transitions for the recorded sequence.

Recently, Moision et al. [8] and Karabed et al. [6] used error-event analysis on partial-response channels to design codes that forbid the appearance of certain patterns in a periodic manner. The resulting codes successfully eliminated problematic sequences in the data recording or retrieval process, and gave improved code distance and detection performance. These codes are known as "distance-enhancing constrained codes." In particular, it was observed that certain time-varying MTR (TMTR) constraints were not only distance-enhancing,

Manuscript received July 22, 2005; revised June 7, 2006. The material in this paper was presented in part at the IEEE International Symposium on Information Theory Adelaide, Australia, Sep. 2005.

T. L. Poo was with the Department of Electrical Engineering, Stanford University, Stanford, CA 94305 USA. She is now with Marvell Semiconductor Inc., Santa Clara, CA 95054 USA (e-mail: 1poo@marvell.com).

B. H. Marcus is with the Department of Mathematics, University of British Columbia, Vancouver, BC V6T 1Z2, Canada (e-mail: marcus@math.ubc.ca).

Communicated by $\emptyset$. Yterhus, Associate Editor for Coding Techniques.

Color versions of Figs. 9-11 are available online at http://ieeexplore.ieee.org.

Digital Object Identifier 10.1109/TIT.2006.881706

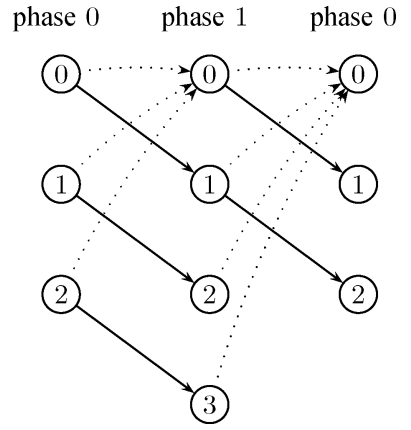

Fig. 1. Standard trellis diagram for the period 2TMTR $(j=2,3)$ constraint.

but had the advantage that they allowed codes of rate $8 / 9$ and above. An example given by Bliss [3], Fitzpatrick and Modlin [5], and Moision et al. [8] is the period $2 \operatorname{TMTR}(j=2,3)$ constraint, which allows a run of two (three) 1 's to end at even (odd) time periods. Its standard trellis diagram is shown in Fig. 1, with solid edges having label 1 and dotted edges having label 0 . This TMTR constraint has been used to construct a rate-8/9 block code that achieves $2.2-\mathrm{dB}$ coding gain on the $\mathrm{E}^{2} \mathrm{PR} 4$ channel in additive-white-Gaussian noise. It was further shown by Moision et al. [8] that this constraint achieved the matched-filter bound (MFB) on the $\mathrm{E}^{n} \mathrm{PR} 4$ channels, with system polynomials $h(D)=(1-D)(1+D)^{n+1}$, for $n=2,3$.

In this paper, we analyze TMTR constraints indexed by a vector $\boldsymbol{m}$, whose coordinates specify periodically the maximum runlengths of 1's ending (or starting) at the positions. We introduce the notion of tightness to classify TMTR $(\boldsymbol{m})$ constraints in Section II and study their minimal graph presentations in Section III. In Section IV, we present new upper bounds on the capacity of TMTR constraints and show that there is a complete linear chain of tight TMTR $(\boldsymbol{m})$ for vectors $\boldsymbol{m}$ up to length 4 ordered by capacity. Finally, in Section V, we show that for MTR constrained systems with unconstrained positions [13], [4], [11], the set of sequences restricted to the constrained positions yields a TMTR constraint. We also derive a new upper bound on the tradeoff function relating the density of unconstrained positions to the maximum code rates. Omitted proofs can be found in [10].

We begin with definitions of the TMTR constraints.

A labeled graph $G=(V, E, L)$ consists of

- a finite set of states $V=V_{G}$;

- a finite set of edges $E=E_{G}$, where each edge $e$ has an initial state $\sigma(e)$ and a terminal state $\tau(e)$, both in $V$;

- an edge labeling $L=L_{G}: E \rightarrow \Sigma$, where $\Sigma$ is a finite alphabet.

Formally, a constrained system or constraint $S=S(G)$ is the set of finite sequences obtained by reading the edge labels of a path in a labeled graph $G$. Such a graph is called a presentation of the constraint. An element in $S$ is called a word. We use $w z$ to denote the concatenation of two words $w$ and $z ;|w|$ denotes the 
length of $w ; w_{i}$ denotes the $i$ th symbol in $w=w_{0} w_{1} \ldots w_{|w|-1}$; $w[x, y]$ denotes the substring of $w$ from positions $x$ to $y$.

Let $\boldsymbol{m}$ be the nonnegative integer vector $\left(m_{0}, \ldots, m_{p-1}\right)$. For all $k \in \mathbb{Z}$, put $m_{k}=m_{i}$ where $i \in\{0, \ldots, p-1\}$ and $k \equiv i$ $(\bmod p)$. From [3], [5], and [8], the $\operatorname{TMTR}(\boldsymbol{m})$ constraint is given as the set of sequences such that at time (or position) $i$, the maximum allowable runlength of 1 's ending at $i$ is $m_{i}$. For this to be a constrained system (see [7]), all shifts of valid sequences have to be included. Let $\sigma^{i}$ denote the cyclic right shift operation on the vector $\boldsymbol{m}$ by $i$ positions. Then

$$
\overline{\operatorname{TMTR}}(\boldsymbol{m}) \triangleq \cup_{i=0}^{p-1} \operatorname{TMTR}\left(\sigma^{i}(\boldsymbol{m})\right)
$$

is a constrained system.

We can also consider the set $\operatorname{TMTR}_{s}(\boldsymbol{m})$ of finite sequences with $m_{i}$ the maximum allowable runlength of 1 's starting at time $i$. Sequences of a fixed length in $\operatorname{TMTR}(\boldsymbol{m})$ and $\operatorname{TMTR}_{s}(\boldsymbol{m})$ are related as follows. For a word $w$ of length $L$, let us write $\overleftarrow{w}$ for the reversal of $w$, i.e., $\overleftarrow{w_{i}}=w_{L-i-1}$ for $0 \leq i \leq L-1$. If $\Omega_{L}$ is a set of words of length $L$, let $\overleftarrow{\Omega}_{L}=\left\{\overleftarrow{w}: w \in \Omega_{L}\right\}$ Then there is a permutation $\boldsymbol{m}^{\prime}=\sigma^{L \bmod p}(\overleftarrow{\boldsymbol{m}})$ such that $\operatorname{TMTR}\left(\boldsymbol{m}^{\prime}\right)_{L}={\overleftarrow{\operatorname{TMTR}_{s}(\boldsymbol{m})}}_{L}$. For the remainder of this paper, we will only deal with $\overline{\text { TMTR }}(\boldsymbol{m})$; dual results hold for $\overline{\mathrm{TMTR}}_{s}(\boldsymbol{m})$

A closely related notion is the TMTR values of a word $w$. Specifically, we define the sequence $r=r(w)$ of instantaneous TMTR values by letting $r_{i}$ be the runlength of 1 's ending at position $i\left(r_{i}=0\right.$ if $\left.w_{i}=0\right)$. Note that if $w$ is contained in TMTR $(\boldsymbol{m})$, then $r_{i} \leq m_{i}$. Conversely, $w$ satisfies $\operatorname{TMTR}(r(w))$.

\section{TIGHT Vectors FOR TMTR $(\boldsymbol{m})$}

Given two vectors $\boldsymbol{m} \neq \boldsymbol{m}^{\prime}$ of the same length, it is possible to have TMTR $(\boldsymbol{m})=\operatorname{TMTR}\left(\boldsymbol{m}^{\prime}\right)$. An example is $\boldsymbol{m}=(2,3,4)$ and $\boldsymbol{m}^{\prime}=(2,3,5)$. To provide a canonical choice for $\boldsymbol{m}$, we introduce the notion of tightness.

As before, fix a vector $\boldsymbol{m}$ of length $p$. Let $\Omega(\boldsymbol{m})$ be the collection of all vectors $\boldsymbol{m}^{\prime}$ of length $p$ such that TMTR $\left(\boldsymbol{m}^{\prime}\right)=$ $\operatorname{TMTR}(\boldsymbol{m})$. Let $\boldsymbol{m}^{*}$ be the component-wise minimum of $\Omega(\boldsymbol{m})$.

\section{Proposition 1: For any $\boldsymbol{m}, \boldsymbol{m}^{*} \in \Omega(\boldsymbol{m})$.}

Proof: Clearly, TMTR $\left(\boldsymbol{m}^{*}\right) \subseteq \operatorname{TMTR}(\boldsymbol{m})$. For the reverse containment, let $w$ be a word in $\operatorname{TMTR}(\boldsymbol{m})$ and fix an arbitrary position $i$. By definition of $\boldsymbol{m}^{*}$, there is some $\boldsymbol{m}^{\prime} \in \Omega(\boldsymbol{m})$ such that $m_{i}^{\prime}=m_{i}^{*}$. Then, $w$ is also contained in $\operatorname{TMTR}\left(\boldsymbol{m}^{\prime}\right)$, so the runlength of 1 's starting at position $i$ in $w$ is at most $m_{i}^{\prime}=$ $m_{i}^{*}$. So $w \in \operatorname{TMTR}\left(\boldsymbol{m}^{*}\right)$. Hence, $\operatorname{TMTR}\left(\boldsymbol{m}^{*}\right)=\operatorname{TMTR}(\boldsymbol{m})$.

We call $\boldsymbol{m}^{*}$ the tight form of $\boldsymbol{m}$. It follows that given two vectors $\boldsymbol{m}, \boldsymbol{m}^{\prime}$ of the same length $p$, TMTR $(\boldsymbol{m})$ coincides with $\operatorname{TMTR}\left(\boldsymbol{m}^{\prime}\right)$ if and only if $\boldsymbol{m}$ and $\boldsymbol{m}^{\prime}$ have the same tight form. However, we need an efficient way to find the tight form $\boldsymbol{m}^{*}$. This can be done using the following necessary and sufficient condition for tightness.

Proposition 2: A vector $\tilde{\boldsymbol{m}} \in \Omega(\boldsymbol{m})$ is the tight form of $\boldsymbol{m}$ if and only if

$$
\tilde{m}_{i+1} \leq \tilde{m}_{i}+1
$$

for all $i \in\{0, \ldots, p-1\}$.
Proof: Suppose $\tilde{m}_{i+1}>\tilde{m}_{i}+1$ for some fixed $i$. Define $\boldsymbol{m}^{\prime}$ by $m_{i+1}^{\prime}=\tilde{m}_{i}+1$ and $m_{k}^{\prime}=m_{k}$ for $0 \leq k \leq p-1, k \neq$ $i+1$. Then, $\boldsymbol{m}^{\prime} \leq \tilde{\boldsymbol{m}}$ componentwise, with strict inequality at $i+1$. So TMTR $\left(\boldsymbol{m}^{\prime}\right) \subseteq \operatorname{TMTR}\left(\boldsymbol{m}^{*}\right)$. Conversely, let $w$ be a word in $\operatorname{TMTR}(\tilde{\boldsymbol{m}})$ and let $k$ be the index of a position in $w$. If $k \equiv i+1(\bmod p)$, then the runlength of 1 's ending at $k$ is at most $\tilde{m}_{i}+1=m_{i+1}^{\prime}$ since the runlength of 1 's ending at $k-1$ is at most $\tilde{m}_{i}$. If $k \not \equiv i+1(\bmod p)$, the runlength of 1 's ending at $k$ is at most $\tilde{m}_{k}=m_{k}^{\prime}$. Thus, $w \in \operatorname{TMTR}\left(\boldsymbol{m}^{\prime}\right)$, showing $\operatorname{TMTR}\left(\boldsymbol{m}^{\prime}\right)=\operatorname{TMTR}(\tilde{\boldsymbol{m}})$. So $\tilde{\boldsymbol{m}}$ is not tight, which proves the necessity of the condition.

For sufficiency, let $\tilde{\boldsymbol{m}}$ satisfy inequality (1). Let $\boldsymbol{m}^{\prime}$ be a vector of the same length as $\tilde{\boldsymbol{m}}$ such that for some component $i_{0}$,

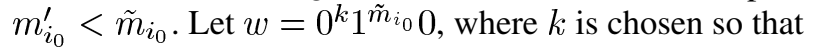

$$
k+\tilde{m}_{i_{0}}+1 \equiv i_{0}(\bmod p) .
$$

It is easily checked that at index $i_{0}$, the runlength of 1 's ending at $i_{0}$ is exactly $\tilde{m}_{i_{0}}$. Then $w$ is contained in TMTR $(\boldsymbol{m})$ by condition (1). However, $w$ is not contained in TMTR $\left(\boldsymbol{m}^{\prime}\right)$ since $m_{i_{0}}^{\prime}<\tilde{m}_{i_{0}}$. This shows that $\boldsymbol{m}^{\prime} \notin \Omega(\boldsymbol{m})$. Therefore, $\tilde{\boldsymbol{m}}$ is tight.

The corresponding tightness condition for $\mathrm{TMTR}_{s}$ is

$$
\tilde{m}_{i-1} \leq \tilde{m}_{i}+1 \text {. }
$$

Using Proposition 2, we give the following algorithm for computing the tight form of a vector $\boldsymbol{m}$. The algorithm cyclically and iteratively reduces each $m_{i}$ until inequality (1) holds for all $i$. Since no component of $\boldsymbol{m}$ ever increases or becomes negative in any iteration and TMTR $(\boldsymbol{m})$ does not change, the algorithm must terminate and output $\boldsymbol{m}^{*}$. In the outline of the algorithm below, the subscripts of the vector $\boldsymbol{m}$ are taken $\bmod p$ and start from 0 . An example illustrating the algorithm is also given.

\section{COMPUTING THE UNIQUE TIGHT $\boldsymbol{m}$ * FROM GIVEN VECTOR $\boldsymbol{m}$}

Input: $m$

$$
\begin{aligned}
& p \leftarrow \text { length }(\boldsymbol{m}) \\
& \text { Count } \leftarrow 1 \\
& k \leftarrow 1 \\
& \text { while Count } \neq p+1 \\
& \text { if } k=1 \\
& \quad \text { Count } \leftarrow 1 \\
& \text { if } \boldsymbol{m}_{k}>\boldsymbol{m}_{k-1}+1 \\
& \quad \boldsymbol{m}_{k} \leftarrow \boldsymbol{m}_{k-1}+1 \\
& \text { else } \\
& \text { Count } \leftarrow \operatorname{Count}+1 \\
& k \leftarrow k+1 \quad(\bmod p)
\end{aligned}
$$

Output: $\boldsymbol{m}$ (which equals $\boldsymbol{m}^{*}$ )

Example 3: Let $\boldsymbol{m}=(5,0,10,3,4,2)$. Then reduce $\boldsymbol{m}$ to its tight form by

$$
\begin{aligned}
(5,0,10,3,4,2) \rightarrow(5,0,1,3,4,2) & \rightarrow(5,0,1,2,4,2) \\
& \rightarrow(5,0,1,2,3,2) \rightarrow(3,0,1,2,3,2) .
\end{aligned}
$$


TABLE I

TIGHT FORMS OF $\boldsymbol{m}$ FOR TMTR FOR $p=2,3,4$

\begin{tabular}{|c|c|}
\hline$p$ & Tight forms \\
\hline \hline 2 & $(s, s),(s, s+1)$ \\
\hline 3 & $(s, s, s),(s, s+1, s+2),(s, s+1, s+1),(s, s, s+1)$ \\
\hline 4 & $(s, s, s, s),(s, s, s, s+1),(s, s, s+1, s+1)$, \\
& $(s, s+1, s, s+1),(s, s, s+1, s+2),(s, s+1, s+1, s+1)$, \\
& $(s, s+1, s+1, s+2),(s, s+1, s+2, s+2)$, \\
& $(s, s+1, s+2, s+3),(s, s+1, s+2, s+1)$ \\
\hline
\end{tabular}

So $\boldsymbol{m}^{*}=(3,0,1,2,3,2)$. Note the necessity of wrapping around at the last step of the reduction.

Finally, the tight forms of $\boldsymbol{m}$ for $p=2,3,4$ are listed (up to their cyclic permutations) in Table I. The number of reduced forms of the TMTR vectors increases exponentially with their length.

\section{GRAPH PRESENTATIONS OF TMTR $(\boldsymbol{m})$}

Let $G$ be a labeled graph. For convenience, we sometimes write

$$
u \stackrel{a}{\longrightarrow} v
$$

to denote an edge or transition from state $u$ to state $v$ with label $a$, and

$$
u \stackrel{L(\eta)}{\rightsquigarrow} v
$$

to denote a path $\eta$ with label $L(\eta)$ from state $u$ to state $v$. A path $\gamma$ of length $l$ in $G$ is a finite sequence of edges $e_{1} e_{2} \ldots e_{l}$ such that $\sigma\left(e_{i+1}\right)=\tau\left(e_{i}\right)$ for $i=1,2, \ldots, l-1$. The state sequence of a path $e_{1} e_{2} \cdots e_{l}$ is the sequence $\sigma\left(e_{1}\right) \sigma\left(e_{2}\right) \cdots \sigma\left(e_{l}\right) \tau\left(e_{l}\right)$. A cycle in $G$ is a path $e_{1} e_{2} \cdots e_{l}$ where $\tau\left(e_{l}\right)=\sigma\left(e_{1}\right)$. A cycle of length 1 is called a loop. A simple cycle is a cycle in which the state sequence consists of distinct states except the initial and terminal states. The period of $G$ is the greatest common divisor of the lengths of cycles in $G$. We say $G$ is deterministic if at each state, all outgoing edges carry distinct labels, and irreducible if for any pair $(u, v)$ of states, there is a path from $u$ to $v$.

For a graph $G$, the adjacency matrix $A=A_{G}$ is the $\left|V_{G}\right| \times$ $\left|V_{G}\right|$ matrix whose entries are indexed by the states of $G$ and $A_{u, v}$ is the number of edges from $u$ to $v$ in $G$.

The follower set of a state $u$ in $G$, denoted by $\mathrm{F}(u)=\mathrm{F}_{G}(u)$, is defined to be the set of all finite words that can be generated from $u$ in $G$. Two states $u$ and $v$ are equivalent if $\mathrm{F}(u)=\mathrm{F}(v)$. The graph $G$ is reduced if no two distinct states in $G$ are equivalent. For a constraint with an irreducible graph presentation, there is a unique minimal (in terms of the number of states) deterministic presentation, called the Shannon cover. It is the unique presentation that is irreducible, deterministic, and reduced. For more background on constrained systems and their presentations, see [7].

\section{A. Standard Trellis Presentation}

A graph presentation of TMTR $(\boldsymbol{m})$ is constructed by concatenating in an appropriate sense the standard presentations of $\operatorname{MTR}\left(m_{i}\right)$ for each $i=0, \ldots, p-1$, and letting the transitions pass from phase $i$ to phase $i+1$. This is known as the standard trellis presentation $G_{T}$. Specifically, the states and transitions of $G_{T}$ are given as follows.

- States: $m_{l}+1$ states in the $l$ th phase, for $0 \leq l \leq p-1$,

$$
\left\{(l, i): 0 \leq l \leq p-1,0 \leq i \leq m_{l}\right\} \text {. }
$$

Here $i$ represents the number of preceding consecutive 1's.

- Transitions: Beginning at phase $l$,

$$
\begin{aligned}
& (l, i) \stackrel{1}{\longrightarrow}((l+1) \bmod p, i+1), \quad \text { if } i<m_{l+1} \\
& (l, i) \stackrel{0}{\longrightarrow}((l+1) \bmod p, 0) .
\end{aligned}
$$

This construction yields $p+\sum_{i=0}^{p-1} m_{i}$ states and takes linear time. Applying the state-merging algorithm [1], which finds and merges equivalent states, we can obtain the Shannon cover for $\overline{\text { TMTR }}(\boldsymbol{m})$. If $\boldsymbol{m}$ is not tight, we can decrease the number of states and transitions in this construction by first reducing $\boldsymbol{m}$ to its tight form.

We say that $\boldsymbol{m}$ has minimal length if there does not exist $\boldsymbol{m}^{\prime}$ of shorter length than $\boldsymbol{m}$ such that TMTR $(\boldsymbol{m})=\operatorname{TMTR}\left(\boldsymbol{m}^{\prime}\right)$. For an equivalence class $\mathcal{C}$ in phase $l$ of $G_{T}$, let $x=\min \{y$ : $(l, y) \in C$; ; we choose $(l, x)$ as the representative of $\mathcal{C}$. We call such points the division points of phase $l$. For $\boldsymbol{m}$ tight with minimal length, the following properties of $G_{T}$ hold.

- Any two states with different phases are not equivalent.

- Two states $(l, x)$ and $(l, y)$ (with $x<y$ ) in the same phase $l$ are not equivalent if and only if there exists an integer $i \geq 1$ such that $x \leq m_{l+i}-i<y$.

- The division points for phase $l$ are given by the set of states $(l, x)$ with

$$
\begin{aligned}
& x \in\left\{\left(m_{l+i}+1-i-k p\right)^{+}:\right. \\
&0 \leq i \leq p-1,0 \leq k \leq\lceil\max (\boldsymbol{m}) / p\rceil\}
\end{aligned}
$$

(where the notation $(z)^{+}=z$ if $z \geq 0$ and 0 if $z<0$ ). The equivalence class of a division point $(l, y)$ consists of the states $\{(l, y),(l, y+1), \ldots,(l, z-1)\}$ where $(l, z)$ is another division point or the state $\left(l, m_{l}\right)$.

This leads to the following explicit description of the Shannon cover.

Proposition 4: Let $\boldsymbol{m}$ be tight with minimal length. Then the states of the Shannon cover of TMTR $(\boldsymbol{m})$ are the division points of all phases in $G_{T}$ as specified above.

Proof: We prove the three properties of $G_{T}$.

1) Assume (for contradiction) that the two states $(l, x)$ and $\left(l^{\prime}, x^{\prime}\right)$ with $l \neq l^{\prime}$ are equivalent. Then $\mathcal{F}(l, x)=\mathcal{F}\left(l^{\prime}, x^{\prime}\right)$ and so $\mathcal{F}(l+1,0)=\mathcal{F}\left(l^{\prime}+1,0\right)$. However, $\mathcal{F}(l+1,0)$ is a TMTR constraint with vector

$$
\boldsymbol{m}_{1}=\left(m_{l+1}, m_{l+2}, \ldots, m_{p-1}, m_{0}, \ldots, m_{l}\right)
$$

while $\mathcal{F}\left(l^{\prime}+1,0\right)$ is a TMTR constraint with vector

$$
\boldsymbol{m}_{2}=\left(m_{l^{\prime}+1}, m_{l^{\prime}+2}, \ldots, m_{p-1}, m_{0}, \ldots, m_{l^{\prime}}\right) .
$$

Thus, $\operatorname{TMTR}\left(\boldsymbol{m}_{1}\right)=\operatorname{TMTR}\left(\boldsymbol{m}_{2}\right)$.

The shifted vectors

$$
\left(m_{l+1}, m_{l+2}, \ldots, m_{l}\right) \text { and }\left(m_{l^{\prime}+1}, m_{l^{\prime}+2}, \ldots, m_{l^{\prime}}\right)
$$

are tight since $\boldsymbol{m}$ is tight by assumption. Then $\sigma^{l+1}(\boldsymbol{m})=$ $\sigma^{l^{\prime}+1}(\boldsymbol{m})$, which implies that $\sigma^{k}(\boldsymbol{m})=\boldsymbol{m}$ for $k=l^{\prime}-l, 1 \leq$ $k \leq p-1$. This in turn implies that

$m_{0} \cdots m_{p-1} m_{0} \cdots m_{p-1} \cdots=m_{0} \cdots m_{k-1} m_{0} \cdots m_{k-1} \cdots$ so TMTR $(\boldsymbol{m})=\operatorname{TMTR}\left(m_{0}, \ldots, m_{k-1}\right)$, contradicting the assumption that $\boldsymbol{m}$ was chosen to be of minimal length.

2) Assume that $(l, x)$ and $(l, y)$ are not equivalent. Then $\mathcal{F}(l, y)$ is a proper subset of $\mathcal{F}(l, x)$. Let $w$ be a word in $\mathcal{F}(l, x)$ which is not contained in $\mathcal{F}(l, y)$. The word $w$ can be taken to be of the form $1^{i}$. Then $x+i \leq m_{l+i}$ (since $1^{i} \in \mathcal{F}(l, x)$ ) 
and $y+i>m_{l+i}$ (since $1^{i} \notin \mathcal{F}(l, y)$ ). This implies that $x \leq m_{l+i}-i<y$.

Conversely, suppose there is an integer $i \geq 1$ such that $x \leq$ $m_{l+i}-i<y$. Then $w=1^{i}$ can be generated from state $(l, x)$ but not from state $(l, y)$. This implies that $\mathcal{F}(l, x) \neq \mathcal{F}(l, y)$, and so the states $(l, x)$ and $(l, y)$ are not equivalent.

3 ) For any phase $l$, we can enumerate the set

$$
\left\{m_{l+i}-i \in\left[0, m_{l}\right]: i \in Z^{+}\right\}
$$

as $\left\{v_{1}, v_{2}, \ldots, v_{k}\right\}$, with $v_{1}<v_{2}<\cdots<v_{k}$. Note that if $v_{i}<x \leq v_{i+1}$, then by part 2$),(l, x)$ is equivalent to $\left(l, v_{i+1}\right)$. Thus, the division points for phase $l$ are given by the set of states $(l, x)$ for $x$ in

$$
\left\{m_{l+i}-i+1 \in\left[0, m_{l}\right]: i \in Z^{+}\right\} \cup\{0\}
$$

(since the state $(l, 0)$ is always a division point). Note that if $\boldsymbol{m}$ is tight, then $m_{l+i}-i \leq m_{\boldsymbol{l}}$. Writing $i=i^{\prime}+k p$, where $0 \leq i^{\prime} \leq p-1$ and $0 \leq k \leq\lceil\max (\boldsymbol{m}) / p\rceil$, we can write the set of division points in the claimed form.

Using part 1), it is clear that the division points in each of the $p$ phases are distinct. Thus, they constitute the states of the Shannon cover of TMTR $(\boldsymbol{m})$ (if merged into those representative classes).

Proposition 5: Let $\boldsymbol{m}$ be a tight vector of minimal length $p$. Then the Shannon cover of TMTR $(\boldsymbol{m})$ has period $p$.

Proof: Any cycle in the trellis presentation $G_{T}$ must have length that is a multiple of $p$. To show this, let $\gamma$ be a cycle of length $l$ in the Shannon cover. This induces a path $\tilde{\gamma}$ of length $l$ (not necessarily a cycle) in $G_{T}$. The initial and terminal states of $\tilde{\gamma}$ are equivalent since $\gamma$ is a cycle, so their phases are the same, by part 1) of the proof of Proposition 4. It follows that $l$ is a multiple of $p$.

To show that the period of the Shannon cover is exactly $p$, we show that there is a cycle of length exactly $p$. By part 3 ) of the proof of Proposition 4, the set of states $\{(l, 0): 0 \leq p-1\}$ in $G_{T}$ are always division points (and hence states in the Shannon cover of TMTR $(\boldsymbol{m})$ ). By construction, there is a cycle of length exactly $p$ in the Shannon cover given by

$$
(0,0) \stackrel{0}{\longrightarrow}(1,0) \stackrel{0}{\longrightarrow}(2,0) \stackrel{0}{\longrightarrow} \cdots \stackrel{0}{\longrightarrow}(p-1,0) \stackrel{0}{\longrightarrow}(0,0) .
$$

\section{B. Shannon Cover When $\boldsymbol{m}=(M-p+1, M-p+2, \ldots, M)$}

Let $\boldsymbol{m}=(M-p+1, M-p+2, \ldots, M)$, that is, $\boldsymbol{m}$ satisfies the tightness condition (1) with equality except for the rightmost coordinate. This includes the TMTR $(2,3)$ constraint mentioned in the Introduction. Construct the graph $G(M, p)$ with exactly $M+1$ states as follows.

- States of $G(M, p)$ : The states are labeled $0,1, \ldots, M$.

- Transitions of $G(M, p)$ : The transitions are given by

$$
\begin{array}{ll}
s \stackrel{1}{\longrightarrow} s+1, & \text { for } s=0,1, \ldots, M-1 \\
s \stackrel{0}{\longrightarrow}(s+1) \bmod p, & \text { for } s=0,1, \ldots, M .
\end{array}
$$

Note that $G(M, p)$ is irreducible and deterministic. Moreover, it is reduced since for any state $s$, the follower set $\mathcal{F}_{G}(s)$ contains the word $1^{M-s}$ but not $1^{M-s+1}$, so the follower sets of the states in $G$ are distinct.

We present a combinatorial proof that $G$ is the Shannon cover of $\overline{\operatorname{TMTR}}(\boldsymbol{m})$.
Proposition 6: The graph $G(M, p)$ is the Shannon cover of $\operatorname{TMTR}(M-p+1, M-p+2, \ldots, M)$.

Proof: We first show that every word $w$ that can be generated from $G$ is contained in TMTR $(\boldsymbol{m})$. Since $G$ is irreducible, without loss of generality, it suffices to consider paths starting at state 0 . Let $s_{0}, s_{1}, s_{2}, \ldots$ be the sequence of states in the path generating $w$

$$
0=s_{0} \stackrel{w_{1}}{\longrightarrow} s_{1} \stackrel{w_{2}}{\longrightarrow} s_{2} \stackrel{w_{3}}{\longrightarrow} \cdots .
$$

By construction, $w$ cannot have a run of more than $M 1$ 's. Note that $s_{i+1} \equiv s_{i}+1 \quad(\bmod p)$ since if $w_{i+1}=1$, then $s_{i+1}=s_{i}+1$ and if $w_{i+1}=0$, then $s_{i+1}=\left(s_{i}+1\right) \bmod p$. Therefore, for all $i \in\{0,1,2, \ldots\}$

$$
s_{i} \equiv i \quad(\bmod p) .
$$

Let $r_{i}$ be the runlength of 1's ending at position $i$ in $w$ (take $r_{i}=0$ if $w_{i}=0$ ). Then $r_{i} \leq s_{i} \leq M$, since for any state $s_{i}$ in $G$, and path $\gamma$ in $G$ terminating at $s_{i}$ such that the label of $\gamma$ is a run of 1 's, the length of $\gamma$ is at most $s_{i}$.

There is a unique element of $\{M-p+1, \ldots, M\}$ that is congruent to $i(\bmod p)$, namely

$$
M-p+1+((i-M-1) \bmod p) .
$$

Equations (2) and (3) together imply

$$
s_{i} \leq M-p+1+((i-M-1) \bmod p)
$$

which yields

$$
r_{i} \leq M-p+1+((i-M-1) \bmod p)
$$

which in turn implies that $w$ satisfies $\overline{\text { TMTR }}(\boldsymbol{m})$.

To prove the converse, let $T=\operatorname{TMTR}\left(\sigma^{i}(M-p+\right.$ $1, \ldots, M)$ ), choosing the shift $i$ such that at position $M$, the TMTR value is $M$. It suffices to show that any word in $T$ can be generated by a path in $G$ starting at state 0 . To do this, let $w$ be a right infinite word which cannot be generated by a path in $G$ starting at state 0 , and show that $w$ violates $T$.

Let $i \geq 0$ be minimal such that $w_{1} \ldots w_{i+1}$ is not the label of a path in $G$ starting at 0 . Let $s_{0}, \ldots, s_{i}$ be the sequence of states for the path $w_{1} \cdots w_{i}$ starting at 0 .

Note that $w_{i+1}=1$ and $s_{i}=M$ since otherwise the path could be extended further. For the case $p=M+1$, it follows immediately that $w$ is not in $T$ since $w_{i+1}=1$ but the TMTR value at $i+1$ is $M-p+1=0\left(\right.$ since $\left.M=s_{i} \equiv i \quad(\bmod p)\right)$. So assume $p<M+1$. Then $w_{i}=1$ since the state $M$ does not have an incoming 0 edge.

So choose $j$ with $0 \leq j<i$ maximal such that $w_{i-j}, w_{i-j+1}, \ldots, w_{i}$ are all 1 's. Then either $s_{i-j-1}=0$ or $s_{i-j-1}$ has an incoming 0 edge, so in either case $s_{i-j-1} \leq p-1$. But then the run of 1's ending at $w_{i}$ has length

$$
j+1=s_{i}-s_{i-j-1} \geq M-(p-1)=M-p+1
$$

so the run of 1's ending at $w_{i+1}$ has length at least $M-p+2$. But the TMTR value at $i+1$ is $M-p+1$ since $M=s_{i} \equiv i$ $(\bmod p)$. Thus, $w$ is not in $T$. 


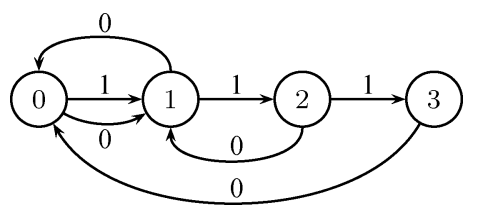

Fig. 2. Shannon cover for $\overline{\mathrm{TMTR}}(2,3)$.

Using Proposition 6, the Shannon cover of $\overline{\text { TMTR }}(2,3)$ consists of four states, and is drawn in Fig. 2. Compare this with its standard trellis presentation in Fig. 1.

The set of sequences in TMTR $(\boldsymbol{m})$ is a constrained system if phase information is included. Its Shannon cover can be obtained from $G(M, p)$ by incorporating phase information into the states of $G(M, p)$ in the following way. Let $(l, i)$ denote a state with phase $l$ and state label $i$. This is achieved by replacing the state label $i$ by $((i+M)(\bmod p), i)$ for each $i=$ $0,1, \ldots, M$. This ensures that a run of $M 1$ 's always terminates at the $(p-1)$ th phase.

We remark that a graph presentation for $\overline{\mathrm{TMTR}}_{s}\left(\boldsymbol{m}^{\prime}\right)$, where $\boldsymbol{m}^{\prime}=\overleftarrow{\boldsymbol{m}}=(M, M-1, \ldots, M-p+1)$ can be obtained by reversing the arrows in the Shannon cover for TMTR $(\boldsymbol{m})$. However, the resulting graph presentation is not necessarily deterministic.

\section{Rome Graphs and Characteristic Equations}

A labeled variable-length graph (VLG) is a labeled graph in which each edge $e$ has length $l(e) \in \mathbb{Z}^{+}$and is labeled by a word $L(e)$ (in some alphabet) of length $l(e)$. A Rome set $\mathcal{R}$ of a labeled graph $G$ is a set of states in $G$ such that every cycle in $G$ passes through a state in $\mathcal{R}$. Trivially, every graph has a Rome set, but we are interested in a Rome set of minimal size. If $G$ has a minimal Rome set with a single element $r$, then we call $G$ a Rome graph and $r$ a Rome state.

The following is a well-known construction of a VLG from an irreducible presentation of a constrained system; see [2].

Let $S$ be a constrained system presented by an irreducible graph $G$ with a Rome set $\mathcal{R}=\left\{r_{1}, \ldots, r_{k}\right\}$. Then there is a VLG $K$ presenting $S$ with vertex set $\mathcal{R}$ and edge set defined as follows. Let $\Gamma$ denote the set of simple paths of first return to $\mathcal{R}$ in $G$, namely, paths whose sequence of states $s_{0}, \ldots, s_{l}$ satisfies $s_{0}, s_{l} \in \mathcal{R}$, and $s_{i} \notin \mathcal{R}$ for $i \neq 0, l$. For each $\gamma \in \Gamma$, with initial state $\alpha$, terminal state $\beta$, and label $L(\gamma)$, there is an edge in $K$ from $\alpha$ to $\beta$ with label $L(\gamma)$.

When the Rome set has size 1, then there is a VLG presentation of $S$ with a single state, whose variable-length edge labels are the labels of simple cycles in $G$.

Proposition 7: Let $\boldsymbol{m}$ be a nonnegative vector of minimal length $p$. Let $d$ denote the minimum number of division points in any phase of the standard trellis presentation of TMTR $(\boldsymbol{m})$. Then the size of the minimal Rome set for the Shannon cover of $\overline{\operatorname{TMTR}}(\boldsymbol{m})$ is at most $\min (p, d)$.

Proof: It is clear that the set of division points in any phase within the trellis presentation constitutes a Rome set. Thus, we can select the smallest set which has size $d$. Also note that the set containing the topmost state in each phase, i.e., $\{(l, 0)$ : $0 \leq l \leq p-1\}$, also forms a Rome set since all cycles in the standard trellis eventually have to return to a state in that set.

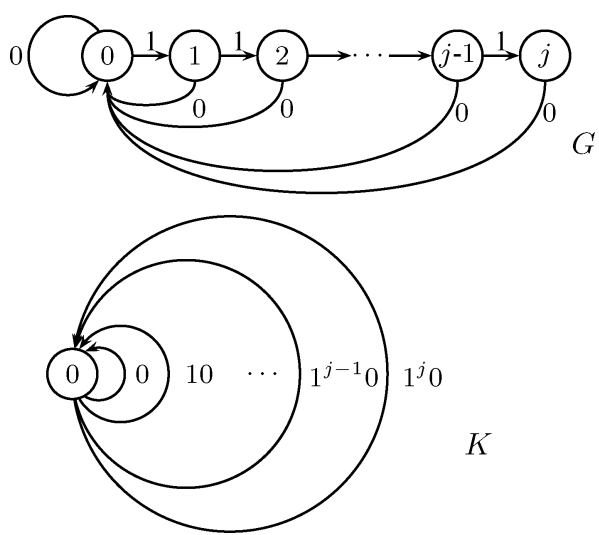

Fig. 3. Shannon cover $G$ of MTR $(j)$ and its variable length presentation $K$.

Thus, the size of the minimal Rome set for the Shannon cover of $\overline{\operatorname{TMTR}}(\boldsymbol{m})$ is at most $\min (p, d)$.

A trivial example where the Shannon cover of $\operatorname{TMTR}(\boldsymbol{m})$ is a Rome graph, is when $m_{k}=0$ for some $0 \leq k \leq p-1$. Then there is only one state in phase $k$ in the trellis presentation $G_{T}$ for TMTR $(\boldsymbol{m})$, so all paths of length at least $p$ in $G_{T}$ must pass through $(k, 0)$. Therefore, all cycles in the Shannon cover of TMTR $(\boldsymbol{m})$ contains $(k, 0)$. Note that the Shannon cover is also a Rome graph whenever the size of the minimal Rome set is 1 .

The edge labels of a VLG can be viewed as distinct symbols (of a new alphabet) of varying time duration (or equivalently symbol length). Shannon [12, Theorem 1] proved the following well-known result.

Theorem 8 ([12]): Let $b_{i j}^{s}$ be the length of the sth edge label from state $i$ to state $j$. Then the capacity of the constrained system is equal to $\log _{2} z$, where $z$ is the largest real root of the determinantal equation:

$$
\left|\sum_{s} z^{-b_{i j}^{s}}-\delta_{i j}\right|=0
$$

where $\delta_{i j}=1$ if $i=j$ and 0 otherwise.

Thus, we can determine the capacity of any constrained system by applying Theorem 8 to a deterministic VLG presentation for the constraint and obtaining an equation whose base- 2 logarithm of its largest positive real root equals the capacity. When the VLG is a Rome graph with period $p$, let $n_{k}$ be the number of simple cycles of length $k p$ for $1 \leq k \leq q$, where $q$ denotes the maximal simple cycle length. Then (4) reduces to

$$
1=\sum_{k=1}^{q} n_{k} z^{-k p}
$$

Example 9: Consider the MTR $(j)$ constraint. Its Shannon cover $G$ is shown in Fig. 3. Clearly, $G$ is a Rome graph with state 0 as the unique Rome state. A VLG presentation $K$ is also depicted in Fig. 3. Clearly, there is one simple cycle each of the lengths $1,2, \ldots, j+1$ starting at the Rome state corresponding to the words $0,10,110, \ldots, 1^{j} 0$. Thus, the determinantal equation of $\operatorname{MTR}(j)$, from (5), becomes $1=\sum_{i=1}^{j+1} z^{-i}$, which can be rearranged to obtain the more commonly seen 
forms: $z^{j+1}=z^{j}+z^{j-1}+\cdots+z+1$, or equivalently, $z^{j+2}-2 z^{j+1}+1=0$.

Proposition 10: The graph $G(M, p)$ is a Rome graph and state $(p-1)$ is a Rome state. Let $(q, r)$ denote the quotient and remainder, respectively, of $M+1$ divided by $p$. There are $2^{p}-1$ simple cycles of length $k p$ for $k=1, \ldots, q-1$, and $2^{p}-2^{p-1-r}$ simple cycles of length $q p$ starting at state $(p-1)$. Thus, the capacity of TMTR $(M-p+1, M-p+2, \ldots, M)$ is equal to $\log z_{0}$, where $z_{0}$ is the largest positive real root of the following equation:

$$
1=\left(2^{p}-2^{p-1-r}\right) z^{-q p}+\left(2^{p}-1\right) \sum_{k=1}^{q-1} z^{-k p} .
$$

Proof: Consider the subgraph $H$ of $G(M, p)$ obtained by deleting the state $p-1$ and all its incoming and outgoing edges. Let $V_{1}=\{0, \ldots, p-2\}$ and $V_{2}=\{p, \ldots, M\}$. Note that the states in $V_{2}$ are inaccessible in $G^{\prime}$ from the states in $V_{1}$, so there is no cycle in $H$ containing states from both $V_{1}$ and $V_{2}$. There is also no cycle in $H$ whose states are all in $V_{1}$, since if there is an edge from $x$ to $y$, with $x, y \in V_{1}$, then $x<y$. Similarly, there is also no cycle in $H$ whose states are all in $V_{2}$. Therefore, $(p-1)$ is a Rome state.

To prove the form of the determinantal equation, call the edges in $G(M, p)$ of the form $s \stackrel{0}{\longrightarrow}(s+1) \bmod p$, where $(s+1) \bmod p \neq s+1$ long edges. Note that each simple cycle in $G(M, p)$ has exactly one long edge. For simplicity, count by assuming that the long edge is used first. Starting at state $s$, with $s \geq p-1$, there are exactly $2^{p-1-t}$ simple cycles starting with a long edge, where $t=(s+1) \bmod p$. The length of all these cycles is

$$
1+(s-t)=s+1-((s+1) \bmod p)
$$

which is clearly a multiple of $p$.

It is easy to check that the set of $s$ satisfying $s+1-$ $((s+1) \bmod p)=k p$ is $\{k p-1, k p, \ldots,(k+1) p-2\}$, and there are exactly $p$ such states. If $M>(k+1) p-2$, then the total number of simple cycles of length $k p$ starting at a state in $\{k p-1, k p, \ldots,(k+1) p-2\}$ is

$$
\sum_{s=k p-1}^{(k+1) p-2} 2^{p-1-(s+1) \bmod p}=\sum_{i=0}^{p-1} 2^{i}=2^{p}-1
$$

which is independent of $k$. Clearly, the largest $k$ for which this holds is $q-1$, where $q$ is the quotient of $M+1$ divided by $p$.

The set of $s$ satisfying $s+1-((s+1) \bmod p)=q p$ is $\{q p-1, q p, \ldots,(q+1) p-2\}$. Since $q p-1 \leq M \leq(q+1) p-2$, this means that the total number of simple cycles of length $q p$ starting at a state satisfying $s+1-((s+1) \bmod p)=q p$ is $\{q p-1, q p, \ldots,(q+1) p-2\}$ is given by a partial sum

$$
\sum_{s=q p-1}^{M} 2^{p-1-(s+1) \bmod p}=\sum_{i=p-1-r}^{p-1} 2^{i}=2^{p}-2^{p-1-r}
$$

where $r$ is the remainder of $M+1$ divided by $p$.

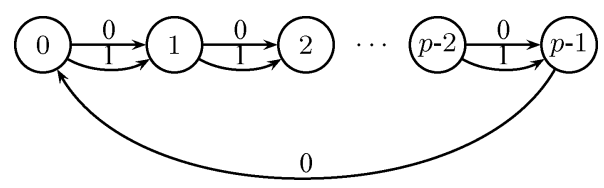

Fig. 4. Shannon cover for $\overline{\operatorname{TMTR}}(0,1, \ldots, p-1)$.

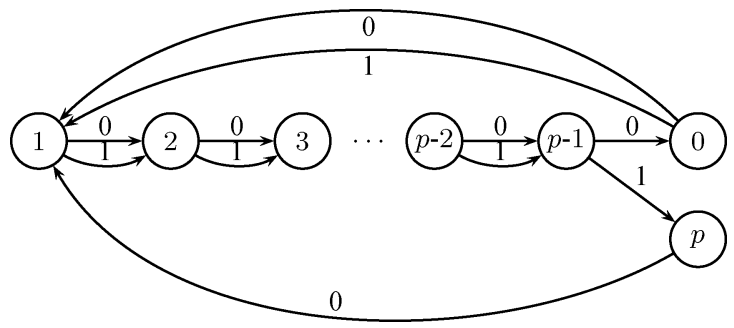

Fig. 5. Shannon cover for $\overline{\operatorname{TMTR}}(1,2, \ldots, p)$.

We remark that if $M \geq 2 p-2$, state $(p-1)$ is the only Rome state in $G(M, p)$ since we can find two cycles whose only common state is state $(p-1)$, namely

$$
0 \stackrel{1}{\longrightarrow} 1 \stackrel{1}{\longrightarrow} 2 \stackrel{1}{\longrightarrow} \cdots \stackrel{1}{\longrightarrow}(p-1) \stackrel{0}{\longrightarrow} 0
$$

and

$$
(p-1) \stackrel{1}{\longrightarrow} p \stackrel{1}{\longrightarrow}(p+1) \stackrel{1}{\longrightarrow} \cdots \stackrel{1}{\longrightarrow}(2 p-2) \stackrel{0}{\longrightarrow}(p-1) .
$$

Example 11: Let $\boldsymbol{m}=(m, m+1)$. From (6), the characteristic equation of $\overline{\text { TMTR }}(m, m+1)$ can be simplified to

$$
\begin{gathered}
z^{m+2}-3 \sum_{k=1}^{\frac{m}{2}} z^{m+2-2 k}-2=0, \quad \text { for } m \text { even } \\
z^{m+2}-3 \sum_{k=0}^{\left\lfloor\frac{m}{2}\right\rfloor+1} z^{m+2-2 k}=0, \quad \text { for } m \text { odd. }
\end{gathered}
$$

Example 12: Let $\boldsymbol{m}=(0,1, \ldots, p-1)$. The Shannon cover of TMTR $(0,1, \ldots, p-1)$ is shown in Fig. 4 , and has exactly $p$ states according to Proposition 6. Clearly, all states are Rome states. There are exactly $2^{p-1}$ simple cycles of length exactly $p$ starting at state 0 , so the characteristic equation is

$$
z^{p}-2^{p-1}=0
$$

and the largest positive real root is $2^{(p-1) / p}$.

Example 13: Let $\boldsymbol{m}=(1,2, \ldots, p)$. The Shannon cover of TMTR $(1,2, \ldots, p)$ is shown in Fig. 5 , and has exactly $p+1$ states by Proposition 6. By inspection, states 1 through $(p-1)$ are all Rome states. There are exactly $2^{p-1}+2^{p-2}$ simple cycles of length $p$ starting at state 1 , so the characteristic equation is

$$
z^{p+1}-\left(2^{p-1}+2^{p-2}\right) z=0
$$

and the largest positive real root is $\left(2^{p-1}+2^{p-2}\right)^{1 / p}$.

We can find TMTR constraints for which their Shannon covers are not Rome graphs. An example is given below.

Example 14: Consider the TMTR $(2,2,3,3)$ constraint, in which the vector $(2,2,3,3)$ is tight. We obtain its Shannon cover 


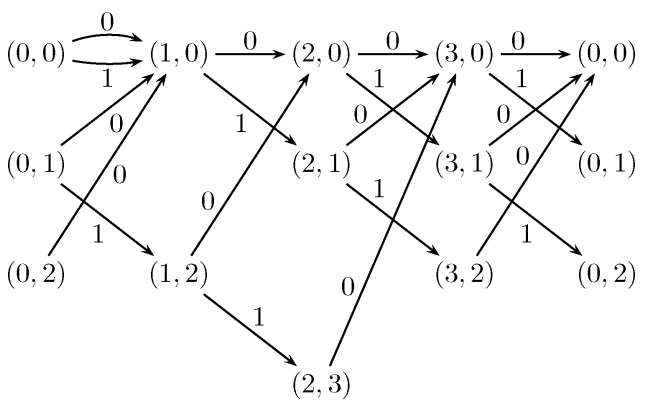

Fig. 6. Shannon cover for $\overline{\operatorname{TMTR}}(2,2,3,3)$.

$G$ by way of the division points in its standard trellis presentation, using Proposition 4. The graph $G$ is shown in Fig. 6 with states in phase 0 repeated for clarity.

Note that $G$ is not a Rome graph because we can find two disjoint simple cycles in $G$, namely

and

$$
(0,0) \stackrel{1}{\longrightarrow}(1,0) \stackrel{0}{\longrightarrow}(2,0) \stackrel{1}{\longrightarrow}(3,1) \stackrel{1}{\longrightarrow}(0,0)
$$

$$
(0,1) \stackrel{1}{\longrightarrow}(1,2) \stackrel{1}{\longrightarrow}(2,3) \stackrel{0}{\longrightarrow}(3,0) \stackrel{1}{\longrightarrow}(0,1) .
$$

Using the adjacency matrix $A$ of $G$, we obtain the characteristic polynomial $\operatorname{det}(\lambda I-A)=\lambda^{11}-13 \lambda^{7}+4 \lambda^{3}$, which can be further factored as $\lambda^{3}\left(\lambda^{4}-3 \lambda^{2}-2\right)\left(\lambda^{4}+3 \lambda^{2}-2\right)$.

It is easily checked that the roots of the characteristic equation are given analytically as follows:

$$
\begin{aligned}
\left\{0,0,0, \pm \sqrt{-\frac{3}{2}+\frac{\sqrt{17}}{2}}, \pm i \sqrt{-\frac{3}{2}+\frac{\sqrt{17}}{2}},\right. \\
\left.\quad \pm \sqrt{\frac{3}{2}+\frac{\sqrt{17}}{2}}, \pm i \sqrt{\frac{3}{2}+\frac{\sqrt{17}}{2}}\right\}
\end{aligned}
$$

and that the largest positive real root is obtained from the factor $\lambda^{4}-3 \lambda^{2}-2$.

In general, for any TMTR $(\boldsymbol{m})$ constraint with a non-Rome Shannon cover, we can use its standard trellis presentations to compute (4) by selecting a Rome set $\mathcal{R}$ consisting of the $p$ states $\{(l, 0): 0 \leq l \leq p-1\}$. The entry $b_{i j}^{s}$ in Theorem 8 would then correspond to the length of a path of first return originating at state $i$ and terminating in state $j$, where $i, j \in \mathcal{R}$, and whose path label is $s$.

\section{BOUNDS ON THE CAPACITY OF TMTR CONSTRAINTS}

In this section, we present some new bounds on the capacity of TMTR constraints. As before, let $\boldsymbol{m}$ be a vector of length $p$ and let $\bar{m}=\sum_{i=0}^{p-1} m_{i} / p$. The capacity of a constrained system $S$, which we denote by $\operatorname{cap}(S)$, is defined by

$$
\operatorname{cap}(S)=\lim _{q \rightarrow \infty} \frac{\log N_{S}(q)}{q}
$$

where $N_{S}(q)$ is the number of words of length $q$ in $S$. Let $G$ be an irreducible deterministic presentation of $S$. The capacity can be computed by $\operatorname{cap}(S)=\log \lambda\left(A_{G}\right)$, where $\lambda(A)$ denotes the largest positive real eigenvalue of $A$.

To simplify notation, let $C(\boldsymbol{m})$ denote the capacity of the $\overline{\text { TMTR }}(\boldsymbol{m})$ constraint. We present new upper bounds on $C(\boldsymbol{m})$ in the next three theorems, whose proofs are given in the Appendix . The first shows that modifying $\boldsymbol{m}$ by adding 1 to the minimum entry and subtracting 1 from the maximum entry does not decrease capacity.

Theorem 15: Let $\boldsymbol{m}$ be a vector with $\max (\boldsymbol{m}) \geq \min (\boldsymbol{m})+2$. Let $i_{1}<i_{2}$ be a pair of positions such that $m_{i_{1}}=\min (\boldsymbol{m})$, $m_{i_{2}}=\max (\boldsymbol{m})$, and $\min (\boldsymbol{m})<m_{i}<\max (\boldsymbol{m})$ for $i_{1}<$ $i<i_{2}$ (performing a cyclic shift of $\boldsymbol{m}$ if necessary). Let $\boldsymbol{m}^{\prime}$ be obtained from $\boldsymbol{m}$ by putting $m_{i_{1}}^{\prime}=m_{i_{1}}+1$ and $m_{i_{2}}^{\prime}=m_{i_{2}}-1$, and $m_{i}^{\prime}=m_{i}$ otherwise. Then $C(\boldsymbol{m})<C\left(\boldsymbol{m}^{\prime}\right)$.

Proof: Let $M=\max (\boldsymbol{m})$ and $s=\min (\boldsymbol{m})$. Let $l_{0}$ be the smallest integer such that $l_{0} p \geq s+1$. We first show that for any integer $k \geq 1$, there is a one-to-one mapping $\phi$ from strings of length $k p$ in TMTR $(\boldsymbol{m})$ into strings of length $\left(k+l_{0}\right) p$ that satisfy TMTR $\left(\boldsymbol{m}^{\prime}\right)$.

Let $w$ be a string of length $k p$ satisfying TMTR $(\boldsymbol{m})$. Let the position indices for $w$ range from 0 to $k p-1$. Let $v=i_{1}+l p$ and $u=i_{2}+l p$ for all $l=0, \ldots, k-1$. Thus, $m_{v}=s$ and $m_{u}=M$. The image sequence $w^{\prime}$ of length $\left(k+l_{0}\right) p$ is indexed from $-l_{0} p$ to $k p-1$. Let $r$ denote the TMTR values of the word $w$, so $r_{i} \leq m_{i}$ for all $0 \leq i \leq k p-1$. Similarly, let $r^{\prime}$ denote the TMTR values of the image sequence $w^{\prime}$. The mapping $\phi$ is constructed so that $r_{v}^{\prime}=s+1$ and $r_{u}^{\prime} \leq M-1$ for all $v$ and $u$, and $r_{i}^{\prime} \leq m_{i}$ for all other position indices $i$. This is depicted for one pair of $(v, u)$ as follows.

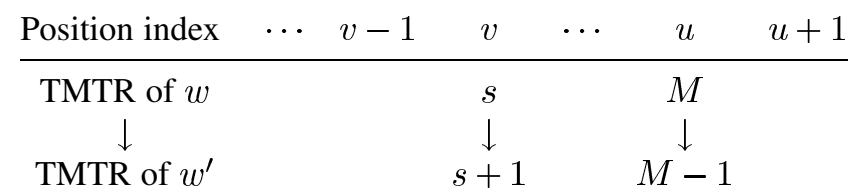

First, we will define the mapping $\phi_{l}$ which maps strings of length $\left(k+l_{0}\right) p$ satisfying TMTR $\left(\left(\boldsymbol{m}^{\prime}\right)^{l+l_{0}} \boldsymbol{m}^{k-l}\right)$ to strings of the same length satisfying TMTR $\left(\left(\boldsymbol{m}^{\prime}\right)^{l+l_{0}+1} \boldsymbol{m}^{k-(l+1)}\right)$ for the $l$ th $(v, u)$ pair, and show that $\phi_{l}$ is one-to-one. The overall mapping $\phi$ is then defined as

$$
\phi=\phi_{k-1} \circ \cdots \circ \phi_{0} \circ \psi
$$

where $\psi$ is the mapping that prepends $l_{0} p$ zeros to a given sequence of length $k p$. Since $\psi$ and $\phi_{l}$ for $l=0, \ldots, k-1$ are one-to-one mappings, it will follow that $\phi$ is also one-to-one.

Given a string $w$ of length $\left(k+l_{0}\right) p$ (indexed from $-l_{0} p$ to $k p-1)$ satisfying $\operatorname{TMTR}\left(\left(\boldsymbol{m}^{\prime}\right)^{l+l_{0}} \boldsymbol{m}^{k-l}\right)$, define $\phi_{l}(w)=$ $w^{\prime}$ as follows. First let $z$ denote the following (possibly empty) substring of $w$ :

$$
z= \begin{cases}w[v-s-1, u-M-1], & \text { if } M=s+2 \text { or } \\ & u-M-1<v+1 \\ w[v-s-1, v+1], & \text { if } M \geq s+3 \text { and } \\ & u-M-1 \geq v+1\end{cases}
$$


Then define the mapping $\phi_{l}$ as follows.

i) If $w$ has $<M 1$ 's ending at $u$, then $w^{\prime}=\phi_{l}(w)=w$.

ii) If $w$ has exactly $M 1$ 's ending at $u$, there are two cases:

a) if $M=s+2$ or $u-M-1<v+1$, then let $w^{\prime}[v-s-1, u]=01^{s+1} 0 z w[v+2+|z|, u]$ with $w^{\prime}=w$ outside of this interval;

b) if $M \geq s+3$ and $u-M-1 \geq v+1$, then let $w^{\prime}[v-s-1, u]=01^{s+1} 0 w[v+2, u-|z|] z$ with $w^{\prime}=w$ outside of this interval.

This forces exactly $(s+1) 1$ 's ending at position $v$ in the image string $w^{\prime}$, when there are exactly $M 1$ 's ending at position $u$ in string $w$. To preserve the information that is lost due to setting $r_{v}^{\prime}=s+1$, the string $z$ is mapped into the space between positions $v+2$ and $u$. We need to check that $z$ fits in this space.

In case ii) a), $|z|=(u-v)-(M-s)+1 \leq u-v-1$ since $M \geq s+2$. (Equality holds when $M=s+2$.) Thus, $z$ fits between positions $v+2$ and $u$, though not necessarily tight; any extra space is filled in by $w[v+2+|z|, u]$. In case ii) b), $|z|=s+3$. Again, $z$ fits between positions $v+2$ and $u$, since the inequalities $u-M-1 \geq v+1$ and $M \geq s+3$ together imply that $u-v-1 \geq M+1 \geq s+4$.

Next, let $\boldsymbol{n}=\left(\boldsymbol{m}^{\prime}\right)^{\bar{l}+l_{0}+1} \boldsymbol{m}^{k-(l+1)}$. We need to check that under $\phi_{l}, r_{v}^{\prime}=s+1$ and $r_{u}^{\prime} \leq M-1$, and for all other positions $i, r_{i}^{\prime} \leq n_{i}$. In fact, it suffices to show this for $v-s-1 \leq i \leq u$, since $\phi_{l}$ only changes $w^{\prime}$ in this local segment.

In case i), $w^{\prime}=w$. Clearly, $r_{i}^{\prime} \leq n_{i}$ for all $i=-s-1$ to $k p-1$. In particular, $r_{u}^{\prime} \leq M-1$ (by construction) and $r_{v}^{\prime} \leq m+1$ trivially. In case ii), the run of $(s+1) 1$ 's ending at $v$ forces the $r_{v}^{\prime}=s+1=n_{v}$. The 0 label in $w^{\prime}$ at positions $v-s-1$ and $v+1$ imply that $r_{v-s-1}^{\prime}=r_{v+1}^{\prime}=0$, so clearly $r_{v-s-1}^{\prime} \leq n_{v-s-1}$ and $r_{v+1}^{\prime} \leq n_{v+1}$. For positions $v-s-2 \leq$ $i \leq v-1$ in $w^{\prime}$, there are at most $s$ 1's ending at positions $i$, and so $r_{i}^{\prime} \leq s \leq n_{i}$. For the remaining positions from $v+2$ to $u$, we need to consider cases ii) a) and ii) b) separately.

For case ii) a), first consider the subcase when $M=s+2$ and $u-M-1 \geq v+1$. Here, $|z|=u-v-1$, so $w^{\prime}[v+2, u]=z$. But in $w, z$ ended at position $u-M-1 \geq v+1$. By definition of $s$ and $M, s+1 \leq r_{u-M-1} \leq M-1$, which implies $r_{u}^{\prime} \leq$ $M-1=n_{u}$ since $z$ is mapped into $w^{\prime}$ to end at position $u$. We claim that for positions $v+2 \leq i \leq u-1, r_{i} \leq n_{i}$. To see this, recall that for $w, r_{i}=s+1=M-1=n_{i}$ for $v+1 \leq i \leq u-1$. It then suffices to show that there is no run of $M 1$ 's ending anywhere between positions $v+2$ and $u-1$ in $w^{\prime}$. Observe that the string $z$ does not contain any run of $M$ 1 's even though the values of $r_{i}$ for $v-s-1 \leq i \leq v-1$ are not known. The length between positions $v-s-1$ and $v$ is exactly $s+2=M$. But $m_{v}=s$, which means that there can be at most $s+11$ 's followed by a zero ending at $v$. For positions $v+1 \leq i \leq u-M-1, r_{i} \leq s+1$, and so there is no run of $M$ 1 's ending anywhere within this interval in $w$. Correspondingly, with $w^{\prime}[v+2, u]=z$ and $w^{\prime}[v+1]=0$, there cannot be a run of $M 1$ 's ending anywhere in the interval $w^{\prime}[v+1, u]$, so $r_{i}^{\prime} \leq n_{i}$ for $v+2 \leq i \leq u-1$.

The next subcase is when $u-M-1<v+1$ and $M=s+2$. Again, $w^{\prime}[v+2, u]=z$. But $u-v-1 \leq M=s+2$. If $u-v-1=M$, rewriting this yields $v=u-M-1$, so $z$ terminates exactly at position $u-M-1$ in $w$, which satisfies $r_{v}=s$. This means that $z$ must contain a zero, so $r_{u}^{\prime} \leq$ $M-1=n_{u}$. Then $w[v+2, u]=1^{u-v-1}$ and $w^{\prime}[v+2, u]=z$ together imply that for positions $v+2 \leq i \leq u-1, r_{i}^{\prime} \leq n_{i}$. If
$u-v-1<M$, then $w^{\prime}[v+1]=0$ eliminates the possibility of a run of $M 1$ 's ending at $u$.

If $u-M-1<v+1$ and $M \geq s+3$, it is easy to verify that

$$
|z|=u-v-(M-s)+1 \leq u-v-2 \leq M-1 .
$$

If $u-M-1 \leq v-1$, then $u-M+1 \leq v+1$, which implies that $w^{\prime}[v+1]=0$ eliminates the possibility of a run of $M 1$ 's ending at $u$ even if $z$ contains all 1 's. Thus, $r_{u}^{\prime} \leq M-1=n_{u}$, and it is clear that $r_{i}^{\prime} \leq n_{i}$ for $v+2 \leq i \leq u-1$. If $u-M-1=v$, then $u-M=v+1$, so $w[v+2, u]=1^{M}$. A run of $M 1$ 's ending in position $u$ in $w^{\prime}$ is not possible since $|z|=s+2$ and $z$ must contain a 0 since $z$ is a substring of $w$ that ends at position $v$ and $r_{v} \leq s$. Thus, $r_{u}^{\prime} \leq M-1=n_{u}$, and $r_{i}^{\prime} \leq n_{i}$ for $v+2 \leq i \leq u-1$.

For case ii) b), when $M \geq s+3$ and $u-M-1 \geq v+1$, then $|z|=s+3 \leq M$. Since $z$ is a substring of $w$ which ended at position $v+1$, where $s+1 \leq r_{v+1} \leq M-1$, $z$ must contain a 0 . When $z$ is mapped to $w^{\prime}$, it is clear that $r_{u}^{\prime} \leq$ $M-1=n_{u}$. The fact that $w[u-|z|+1, u]=1^{|z|-1}$ while $w^{\prime}[u-|z|+1, u]=z$ and $w^{\prime}[u-|z|]=w[u-|z|]=0$ implies that $r_{i}^{\prime} \leq n_{i}$ for $u-|z|+1 \leq i \leq u$. For the remaining positions $v+2 \leq i \leq u-|z|$, it is clear that $r_{i}^{\prime} \leq n_{i}$ since $w^{\prime}[v+2, u-|z|]=w[v+2, u-|z|]$.

To show that $\phi_{l}$ is one-to-one, we show that given a $w^{\prime}$ satisfying TMTR $(\boldsymbol{x})$, there is at most one pre-image $w$ satisfying $\operatorname{TMTR}(\boldsymbol{y})$, where $\boldsymbol{x}=\left(\boldsymbol{m}^{\prime}\right)^{l+l_{0}+1} \boldsymbol{m}^{k-(l+1)}$ and $\boldsymbol{y}=\left(\boldsymbol{m}^{\prime}\right)^{l+l_{0}} \boldsymbol{m}^{k-l}$. The position indices for $w^{\prime}$ and $w$ range from $-l_{0} p$ to $k p-1$. By comparing vectors $\boldsymbol{x}$ and $\boldsymbol{y}$, we can determine the positions $v=i_{1}+l p$ and $u=i_{2}+l p$ where $x_{v}=s+1, y_{v}=s, x_{u}=M-1$, and $y_{v}=M$. We can distinguish between cases i) and ii) by observing if there is a run of $(s+1) 1$ 's ending at position $v$ in $w^{\prime}$. If there is no such run, we are in case i), and $w=w^{\prime}$. Otherwise, we are in case ii), so $w\left[-l_{0} p, v-s-2\right]=w^{\prime}[u+1, k p-1]$. Using the values of $u, v, M, s$, we can further distinguish between cases ii) a) and ii) b) to reconstruct $w$ from positions $v-s-1$ to $u$. Namely, in case ii) a), we can determine $z=w^{\prime}[v+2, v+1+|z|]$, and thus assign $w[v-s-1, u]=z 01^{M}$. In case ii) b), identify the string $z=w^{\prime}[u-|z|+1, u]$, and assign $w[v-s-1, u]=z w^{\prime}[v+2, u-M] 1^{M}$.

The overall mapping $\phi=\phi_{k-1} \circ \cdots \circ \phi_{0} \circ \psi$ is thus one-to-one, mapping strings of length $k p$ satisfying TMTR $(\boldsymbol{m})$ into strings of length $\left(k+l_{0}\right) p$ satisfying TMTR $\left(\boldsymbol{m}^{\prime}\right)$. Thus, letting $N_{S}(l)$ denote the number of strings of length $l$ satisfying $S$, we have

$$
\begin{aligned}
N_{\mathrm{TMTR}(\boldsymbol{m})}(k p) & \leq N_{\mathrm{TMTR}\left(\boldsymbol{m}^{\prime}\right)}\left(k p+l_{0} p\right) \\
& \leq 2^{l_{0} p} N_{\mathrm{TMTR}\left(\boldsymbol{m}^{\prime}\right)}(k p) .
\end{aligned}
$$

Taking log on both sides and dividing by $k p$ yields

$$
\frac{\log N_{\mathrm{TMTR}(\boldsymbol{m})}(k p)}{k p} \leq \frac{l_{0}}{k}+\frac{\log N_{\mathrm{TMTR}\left(\boldsymbol{m}^{\prime}\right)}(k p)}{k p} .
$$

Taking limits as $k \rightarrow \infty$, we obtain $C(\boldsymbol{m}) \leq C\left(\boldsymbol{m}^{\prime}\right)$.

It remains only to show strict inequality. For simplicity, we assume that $s \geq 1$ (the case $s=0$ can be handled by an easier argument). 
There is a word $x=x[-s, u+2]$ in TMTR $\left(\boldsymbol{m}^{\prime}\right)$ such that $x[v-s, v]=1^{s+1}$ and $x[u, u+2]=010$. Let $S$ denote the constrained system obtained by forbidding the word $x$ from the constrained system $S^{\prime}=\overline{\mathrm{TMTR}}\left(\boldsymbol{m}^{\prime}\right)$. Since $S^{\prime}$ is irreducible, any proper subsystem, in particular $S$, of $S^{\prime}$ must have strictly smaller capacity [7]. Thus, to show that the inequality asserted in the theorem is strict, it suffices to show that any word $y$ of length $\left(k+\ell_{0}\right) p$ in the image of $\phi$ must be in $S$. It is enough to show that $y$ does not contain the word $x$ in the sense that for all $\ell=0, \ldots, k-1$, for the $\ell$ th pair $u, v$ it is impossible to have:

$$
y[v-s, v]=1^{s+1} \quad \text { and } \quad y[u, u+2]=010 .
$$

Let $w$ be the pre-image of $y$. We claim that for the $\ell$ th pair $u, v$,

$$
\left(\phi_{\ell-1} \circ \cdots \circ \phi_{0} \psi(w)\right)[v-s, v]=y[v-s, v]
$$

and

$$
\left(\phi_{\ell-1} \circ \cdots \circ \phi_{0} \psi(w)\right)[u, u+2]=y[u, u+2] .
$$

This is equivalent to saying that none of $\phi_{\ell}, \phi_{\ell+1}, \ldots, \phi_{k-1}$ can change any symbol in $[v-s, v]$ or $[u, u+2]$ (again, here $u, v$ denote the $\ell$ th pair). This follows from the facts that 1 ) the string $01^{s+1} 0$ cannot properly overlap itself except at the rightmost coordinate and 2) the string $01^{s+1} 0$ cannot overlap the string 010 except at the rightmost coordinate.

Since $\phi_{\ell-1}$ can produce $1^{s+1}$ ending at the $\ell$ th $v$ only by an application of case ii), it follows that for the $\ell$ th $u, w[u-M+$ $1, u+1]=1^{M+1}$. But this contradicts the fact that $M$ is the maximal TMTR value in $\boldsymbol{m}$.

Let $\bar{m}=\sum_{i=0}^{p-1} m_{i} / p$. Applying Theorem 15 repeatedly to $\boldsymbol{m}$ yields an upper bound based on $\bar{m}$.

Theorem 16: $C(\boldsymbol{m})<\operatorname{cap}(\operatorname{MTR}(\lceil\bar{m}\rceil))$.

Proof: Applying the mapping $\phi$ in the proof of Theorem 15 repeatedly to the vector $\boldsymbol{m}$, we obtain an $\boldsymbol{m}^{\prime}$ with $m_{i}^{\prime}=t$ for all $i$, or $m_{i}^{\prime} \in\{t, t-1\}$ for all $i$, where $t$ is some positive integer. Each time, $\bar{m}^{\prime}=\bar{m}$, so $C(\boldsymbol{m}) \leq C\left(\boldsymbol{m}^{\prime}\right)<\operatorname{cap}(\operatorname{MTR}(t))$, where $t=\lceil\bar{m}\rceil$.

To obtain the best possible bound in Theorem 16, we reduce the vector $\boldsymbol{m}$ to its tight form before applying the theorem.

For TMTR vectors of the form $(M-p+1, M-p+2, \ldots, M)$, a strict upper bound on the capacity can be obtained, which is sharper than the bound of Theorem 16 when $\bar{m}$ is not an integer.

Theorem 17: Let $\boldsymbol{m}=(M-p+1, M-p+2, \ldots, M)$ (with $p \geq 2, \boldsymbol{m} \neq(0,1)$ ), so $\bar{m}=M-(p-1) / 2$. Then $C(\boldsymbol{m})<\log _{2} \lambda(\bar{m})$, where $\lambda(\bar{m})$ is the largest positive real root of the equation

$$
z^{\bar{m}+2}-2 z^{\bar{m}+1}+1=0
$$

(the latter being the characteristic equation for the standard presentation of MTR ( $\bar{m})$ if $\bar{m} \in \mathbb{Z}^{+}$as seen in Example 9). An algebraic proof of the theorem is given in the Appendix. The proof idea is as follows. Let $h(z)=z^{\bar{m}+2}-2 z^{\bar{m}+1}+1$. Let

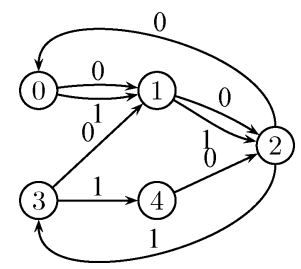

Fig. 7. Shannon cover of TMTR $(2,3,4)$.

$g(z)$ denote the polynomial obtained from (6), such that the capacity of TMTR $(M-p+1, M-p+2, \ldots, M)$ equals the base-2 logarithm of the largest positive real root of $g(z)=0$. To show that the largest positive real root of $h$ exceeds that of $g$ in some interval $[a, b]$, we show that for $\alpha$, the largest real root of $g$ in $[a, b], h(\alpha)<0$. and $h(b)>0$.

Example 18: Let $\boldsymbol{m}=(2,3,4,5)$, whose capacity $C(\boldsymbol{m})=$ 0.9518. Theorem 15 gives $C(\boldsymbol{m})<C(3,3,4,4)=0.9615$, and Theorem 16 implies $C(\boldsymbol{m})<\operatorname{cap}(\operatorname{MTR}(4))=0.9752$, while Theorem 17 yields $C(\boldsymbol{m})<0.9639$.

Example 19: Consider the TMTR $(2,3,4)$ constraint, whose Shannon cover is given by Proposition 6 and depicted in Fig. 7. Its capacity is 0.9358 . Now, $\bar{m}$ is exactly 3 . By Theorem 17 , $C(2,3,4)<\operatorname{cap}(\operatorname{MTR}(3))=0.9468$.

We conjecture the following.

Conjecture 20:

1) For any tight vector $\boldsymbol{m}$ with minimal length $p$

$$
\frac{1}{p} \sum_{i=0}^{p-1} \operatorname{cap}\left(\operatorname{MTR}\left(m_{i}\right)\right)<C(\boldsymbol{m}) .
$$

2) For any tight vector $\boldsymbol{m}$ with minimal length $p$ and $\bar{m} \geq 2$

$$
C(\boldsymbol{m})<\log _{2} \lambda
$$

where $\lambda$ is the largest positive real root of (11).

Note that part 2) of Conjecture 20 is proven in a special case in Theorem 17. Extensive Matlab computations also give credence to the conjecture. We remark that if $\bar{m}$ is an integer, the conjecture would read as follows. For vectors $\boldsymbol{m}$ with $\bar{m} \geq 2$

$$
\frac{1}{p}\left(\sum_{i=0}^{p-1} \operatorname{cap}\left(\operatorname{MTR}\left(m_{i}\right)\right)\right)<C(\boldsymbol{m})<\operatorname{cap}(\operatorname{MTR}(\bar{m})) .
$$

Note that the expression on the left is at most that on the right due to strict concavity of the largest positive real root of (11). The latter fact can be derived using [4, Lemma 2], which uses differentiation techniques on (11). We include the derivation below. Let $\lambda_{j}$ denote the largest positive real eigenvalue of the adjacency matrix of the standard presentation of MTR $(j)$. It is well known that $\operatorname{cap}(\operatorname{MTR}(j))=\log \lambda_{j}$. From [4, Lemma 2], the function $k \mapsto k \log \left(\lambda_{k-1}\right)$ is strictly concave on the domain of positive integers.

This implies that

$$
\lambda_{k+1}\left(\frac{\lambda_{k+1}}{\lambda_{k}}\right)^{k+1} \leq \lambda_{k}\left(\frac{\lambda_{k}}{\lambda_{k-1}}\right)^{k} .
$$


But observe that we can rewrite the left-hand side as

$$
\frac{\lambda_{k+1}^{2}}{\lambda_{k}}\left(\frac{\lambda_{k+1}}{\lambda_{k}}\right)^{k}
$$

which in turn implies

$$
\left(\frac{\lambda_{k+1}}{\lambda_{k}}\right)^{k} \leq\left(\frac{\lambda_{k}}{\lambda_{k-1}}\right)^{k}
$$

Taking the $k$ th root on both sides yields the desired inequality

$$
\lambda_{k+1} \lambda_{k-1} \leq \lambda_{k}^{2} .
$$

This shows that the function $k \mapsto \log \lambda_{k}$ is strictly concave on the domain of positive integers.

We also remark that part 2) of Conjecture 20 would follow from Theorem 15 if part 2) is proven in the special case when $\max (\boldsymbol{m})=\min (\boldsymbol{m})+1$.

\section{A. Complete Linear Ordering of $C(\boldsymbol{m})$ Up to Period 4}

It is difficult to compare the capacity of TMTR constraints of different lengths, unless one constraint is contained in the other. Generally, TMTR constraints are not determined by their capacities as there can be different tight TMTR vectors $\boldsymbol{m}_{1}$ and $\boldsymbol{m}_{2}$ for which $C\left(\boldsymbol{m}_{1}\right)=C\left(\boldsymbol{m}_{2}\right)$. We give an example of this below.

Example 21: Consider the period $2 \overline{\mathrm{TMTR}}(2,3)$ constraint, and the period $4 \overline{\text { TMTR }}(2,2,3,3)$ constraint. Both vectors satisfy (1), and are thus tight.

Neither of these two constraints is contained in the other. For example, the word 0110111001110 satisfies TMTR $(2,2,3,3)$ but not $\overline{\operatorname{TMTR}}(2,3)$, while the word 01110111000111 satisfies $\operatorname{TMTR}(2,3)$ but not $\overline{\operatorname{TMTR}}(2,2,3,3)$.

From (7), the characteristic polynomial for $\overline{\operatorname{TMTR}}(2,3)$ is $\lambda^{4}-3 \lambda^{2}-2=0$, whose roots are

$$
\pm \sqrt{\frac{3}{2}+\frac{\sqrt{17}}{2}}, \quad \pm i \sqrt{-\frac{3}{2}+\frac{\sqrt{17}}{2}} .
$$

From Example 14, the largest positive real root of the characteristic equation of TMTR $(2,2,3,3)$ is also $\sqrt{\frac{3}{2}+\frac{\sqrt{17}}{2}}$. Hence, $\overline{\operatorname{TMTR}}(2,3)$ and $\overline{\text { TMTR }}(2,2,3,3)$ have the same capacity.

We can further establish the following capacity relations.

Proposition 22: For any positive integer $t$

1) a) $C(t, t+1)=C(t, t, t+1, t+1)$ for $t \equiv 2(\bmod 4)$;

b) $C(t, t+1)>C(t, t, t+1, t+1)$ for $t \equiv 0(\bmod 4)$;

c) $C(t, t+1)<C(t, t, t+1, t+1)$ for $t \equiv 1,3$ $(\bmod 4)$.

2) $C(t, t+1)<C(t, t, t+1, t+2)$ for $t \equiv 0(\bmod 4)$.

3) $C(t, t+1, t+2, t+1)=C(t, t+1, t+1, t+2)$.

4) $C(t, t+1, t+2, t+2)>\operatorname{cap}(\operatorname{MTR}(t+1))$ for $t+1 \not \equiv$ $0 \bmod 4$ and $C(t, t+1, t+2, t+2)<\operatorname{cap}(\operatorname{MTR}(t+1))$ when $t+1 \equiv 0(\bmod 4)$.

5) $\operatorname{cap}(\operatorname{MTR}(t+1))<C(t, t+1, t+2, t+3)$.

6) $C(t, t+1, t+2, t+3)<C(t+1, t+1, t+1, t+2)$.
The proofs of the various inequalities in Proposition 22 are very similar, so we shall only prove part 1) in detail in the Appendix . There are two steps, the first being to derive the characteristic equation of the TMTR $(\boldsymbol{m})$ constraint using Theorem 8. This involves constructing the VLG of its standard trellis presentation by selecting $|\boldsymbol{m}|$ trellis states, namely, $\{(0,0),(1,0), \ldots,(m, 0)\}$ as the Rome set. For a given congruence class of $\boldsymbol{m}$, counting the paths and path lengths of first return to the Rome set enables us to derive the matrix whose characteristic equation is described by (4).

Once the characteristic equation of the constraint is determined, the second step uses algebraic techniques similar to that of the proof of Theorem 17.

Using Proposition 22 and Theorems 15 and 17, we give an explicit linear ordering of the TMTR $(\boldsymbol{m})$ constraints by capacity for tight $\boldsymbol{m}$ of lengths $p=2,3$, and 4 . Let $t$ be a positive integer. For $p=2$

$$
C(t, t)<C(t, t+1)<C(t+1, t+1) .
$$

For $p=3$,

$$
\begin{aligned}
C(t, t, t) & <C(t, t, t+1) \\
& <C(t, t+1, t+1) \\
& <C(t, t+1, t+2) \\
& <C(t+1, t+1, t+1)
\end{aligned}
$$

where the last strict inequality is due to Theorem 17.

For $p=4$

$$
\begin{aligned}
& C(t, t, t, t) \\
& \quad<C(t, t, t, t+1) \\
& \quad<C(t, t, t+1, t+1) \stackrel{(\text { a) }}{\gtrless} C(t, t+1, t, t+1) \\
& \quad \stackrel{\text { (b) }}{<} C(t, t, t+1, t+2) \\
& \quad \stackrel{\text { (c) }}{<} C(t, t+1, t+1, t+1), \\
& \quad<C(t, t+1, t+1, t+2) \stackrel{(\mathrm{d})}{=} C(t, t+1, t+2, t+1) \\
& \quad<C(t, t+1, t+2, t+2) \stackrel{(\mathrm{e})}{\gtrless} C(t+1, t+1, t+1, t+1) \\
& \quad \stackrel{(\mathrm{f})}{<} C(t, t+1, t+2, t+3) \\
& \quad \stackrel{\text { g })}{<} C(t+1, t+1, t+1, t+2)
\end{aligned}
$$

where steps (a), (b), (d), (e), (f), and (g) are due to Proposition 22 parts 1), 2), 3), 4), 5), and 6), respectively, while step (c) is due to Theorem 15. In particular, note that in step (e), even though $C(t+1, t+1, t+1, t+1)$ may be strictly greater or less than $C(t, t+1, t+2, t+2)$ depending on the equivalence class of $t \bmod 4, C(t+1, t+1, t+1, t+1)$ still strictly exceeds $C(t, t+1, t+2, t+1)$ (in the preceding line) due to Theorem 15 . The symbol $(\gtrless)$ indicates that the relation between the two quantities could be $=,>$, or $<(>$ or $<)$ depending on the value of $t \bmod p$. In lines (b) and (f), the quantity on the right of the inequality is strictly greater than all of the quantities above it. 


\section{ON MTR CONSTRAINTS With UNCONSTRAINED POSITIONS}

Let $S$ be a constrained system. For a given period $N$, let $U \subseteq$ $\{0,1, \ldots, N-1\}$. Following the scheme in [4], where error correction parity bits are inserted into prespecified "unconstrained" positions that can freely take on either 0 or 1 without violating the existing modulation constraint, let $U$ denote a set of unconstrained positions. A word $w^{\prime}$ is a $U$-flip of $w$ if $w_{i}^{\prime}=w_{i}$ whenever $i \bmod N \notin U$. The $(U, N)$-unconstrained version of $S$, denoted by $S_{U, N}$, is the set of all sequences $w \in S$ such that $w_{i}=1$ for all $i \bmod N \in U$, and all $U$-flips of $w$ belong to $S$. Let $S_{U, N}(k)$ denote the set of all sequences in $S_{U, N}$ of length $k$. Let $U^{c}$ denote the complement of $U$, and $S^{\prime}$ denote the restriction of $S_{U, N}$ to the constrained positions $U^{c}$. This is a periodic constraint with period $\left|U^{c}\right|$.

The code rate $R$ for a given configuration $(U, N)$ with insertion rate $\rho=|U| / N$ is defined as

$$
R=R\left(\bar{S}_{U, N}\right)=\limsup _{k \rightarrow \infty} \frac{\log \left|S_{U, N}(k)\right|}{k} .
$$

The optimal code rate for a given $\rho$ is

$$
f_{S}(\rho)=\limsup _{N \rightarrow \infty} \max _{U \subseteq\{0, \ldots, N-1\}:|U| / N=\rho} \frac{\log \left|S_{U, N}(N)\right|}{N} .
$$

For finite-type constraints, which includes MTR $(j)$ constraints, $f_{S}$ can be achieved by periodic $(U, N)$ configurations (see [11]). We call $f_{S}$ the insertion-rate-code-rate tradeoff function, or in short, the tradeoff function.

For the rest of this section, let $S=\operatorname{MTR}(j)$. View $U$ as a set of points on a circle of $N$ points. Addition of numbers will proceed clockwise (to the right). For a point $x$ on the circle, let

$$
n(x)=|U \cap[x-(j-s(x)), x]|
$$

where $s$ is the number of consecutive 1's immediately to the right of $x$. Note that when both $x$ and $x+1$ are in $U^{c}$, then $s=0$, and $n(x)=|U \cap[x-j, x]| \leq j$. When $x$ is in $U^{c}$ and $x+1$ in $U$, then $n(x)=|U \cap[x-(j-s), x]| \leq j-s$. Let $g$ denote the one-to-one correspondence from constrained positions indexed $(\bmod N-|U|)$ to positions on the circle in $\left(U^{c} \bmod N\right)$.

Theorem 23: Let $S=\operatorname{MTR}(j)$. Then $S^{\prime}$ is a $\overline{\operatorname{TMTR}}(\boldsymbol{m})$ constraint with tight parameters

$$
m_{i}=j-s(g(i))-n(g(i))
$$

for $i=0, \ldots, N-|U|-1$.

Proof: For a position $i \bmod N-|U|$ for the constraint, let $x$ be the corresponding position on the circle, i.e., $g(i)=x$. We will show that the longest run of 1's (in the constrained positions) ending at $x$ has length $j-s(x)-n(x)$.

Let $I$ denote the interval $[x-(j-s(x)), x+s(x)]$. Then $I$ is a block of length $j+1$ containing $n(x)+s(x)$ unconstrained positions and $j+1-(n(x)+s(x))$ constrained positions.

If all of the $j+1-(n(x)+s(x))$ constrained positions in $I$ were filled in with 1 's, then $I$ would be a block of length $j+1$ with only unconstrained positions and 1's, which violates the constraint. So at least one constrained position in $I$ must be a 0 .
This shows that the longest run of 1's ending at $x$ has length at most $j-(n(x)+s(x))$.

On the other hand, if the first constrained position in $I$ is a 0 and the rest of the constrained positions in $I$ are 1 's, then $I$ satisfies the constraint. Moreover, since the next position after $I$ is constrained (by definition of $s(x)$ ), it is possible to have a run of $j-s(x)-n(x) 1$ 's ending at $x$, by making the position immediately after $I$ a 0 .

To show that $\boldsymbol{m}$ is tight, consider the following. For any $i$, $i+1 \in\{0, \ldots, N-|U|-1\}$, let $x=f(i)$ and $y=f(i+1)$. Let $I_{x}$ denote the interval $[x-(j-s(x)), x+s(x)]$ and $I_{y}$ denote the interval $[y-(j-s(y)), y+s(y)]$. Both $I_{x}$ and $I_{y}$ are of length $j+1$ and share some overlap $I_{x \cap y}$. Let $B(I)$ denote the number of unconstrained positions in an interval $I$. Then the difference in the number of unconstrained positions in $I_{x}$ and $I_{y}$ is equal to the difference in the number of unconstrained positions in $I_{x} \backslash I_{x \cap y}$ and $I_{y} \backslash I_{x \cap y}$, i.e.,

$$
\begin{aligned}
B\left(I_{x}\right)-B\left(I_{y}\right)= & B([x-(j-s(x)), y-(j-s(y))-1]) \\
& -B([x+s(x)+1, y+s(y)]) \\
\leq & |[x-(j-s(x)), y-(j-s(y))-1]|-s(y) \\
\leq & y-x+s(y)-s(x)-s(y) \\
\leq & y-x-(y-x-1) \\
\leq & 1
\end{aligned}
$$

since $s(x)=y-x-1$. This shows that $m_{i+1} \leq m_{i}+1$ for all $i \in\{0, \ldots, N-|U|-1\}$.

Example 24: Let $S=\operatorname{MTR}(3)$. Let insertion rate $x=4 / 9$, block length $N=9$, and $U=\{1,2,3,6\}$. Then from Theorem $23, S^{\prime}=\overline{\operatorname{TMTR}}(0,0,1,2,2)$.

Corollary 25: $R\left(S_{U, N}\right)=(1-|U| / N) \operatorname{Cap}_{\overline{\mathrm{TMTR}}}(\boldsymbol{m})$, where $\boldsymbol{m}$ is obtained from Theorem 23 .

\section{A. New Upper and Lower Bounds for the Tradeoff Function for MTR Constraints}

Let $A$ denote the adjacency matrix for the $\operatorname{MTR}(j)$ constraint. Let $B$ be the matrix obtained from $A$ by replacing all entries of the first column by zeros. Given a length $N$, a parity insertion rate $x \in[0,1]$, and a specification of unconstrained positions $U \subseteq\{0, \ldots, N-1\}$ with $|U|=k$ and $k / N=x$, define the matrix

$$
M=\prod_{i=0}^{N-1} M_{i}
$$

where $M_{i}=B$ if $i \in U$ and $M_{i}=A$ if $i \notin U$. Then each entry of $M$ represents the number of sequences in $S_{U, N}$ that begin with a restricted set of prefixes and suffixes. We can view $M$ as a sequence of $A$ 's and $B$ 's, which can be broken up into consecutive runs of $A$ 's followed by runs of $B$ 's. Note that if $M \neq 0$, the run of $B$ 's cannot exceed length $j$ since $B$ is nilpotent $\left(B^{j+1}=0\right)$, but the run of $A$ 's can be of any length (less than the block length $N)$.

Define the VLG $H=H_{j}$ whose vertex set of size $2^{j-1}+1$ consists of all $j$-tuples over the alphabet $\{A, B\}$ ending in $B$ as well as the $j$-tuple $A^{j}$. For every vertex $v$ and each $L=A^{r} B^{s}$ 
with $1 \leq r \leq j$ and $0 \leq s \leq j$, endow $H$ with an edge labeled $L$ from a vertex $v$ to one labeled by the suffix of length $j$ of $v L$. In particular, there is an edge labeled $A^{j}$ from every state ending in $B$ to the state $A^{j}$ and a self-loop labeled $A$ at state $A^{j}$. Clearly, $H$ has parallel edges with distinct variable-length edge labels, so $H$ is deterministic. Self-loops in $H$ are treated as simple cycles of length 1 .

Traversing a path $\pi$ in $H$, we can read off the edge labels to obtain the path label $w(\pi)$, which is a sequence of $A$ 's and $B$ 's. Alternatively, we can think of the $A$ 's as constrained positions and the $B$ 's as unconstrained positions, where at the constrained positions, we can determine from Theorem 23 the TMTR values using a sliding window of length $j+1$ and counting the number of unconstrained positions within that window.

A special feature of $H$ is that each edge in $H$ yields a definite sequence (or vector) of TMTR values. This is because a particular edge $e$, together with its starting and terminal state, has sufficient information in its edge label $L(e)=\left(A^{x}, B^{y}\right)$ and starting state label to determine the TMTR values at the positions of the $x A$ 's. Thus, for two connected simple cycles $\gamma_{1}, \gamma_{2}$ in $H$ with TMTR vectors $\boldsymbol{m}_{1}$ and $\boldsymbol{m}_{2}$, respectively, the TMTR vector for the combined cycle $\gamma_{1} \gamma_{2}$ is simply $\boldsymbol{m}_{1} \boldsymbol{m}_{2}$. Recall that any cycle can be decomposed into a collection of simple cycles. This special property of $H$ imposes a linear relationship between TMTR values on a cycle and those on the simple cycles, even though there is not necessarily any corresponding connection between the largest positive real eigenvalue of the $A, B$ products of the cycle labels.

We also define the insertion rate of a path (or cycle) in $H$ as the proportion of $B$ 's in its path (cycle) label to the length of its path (cycle) label.

We will describe an upper bound on the tradeoff function for $\operatorname{MTR}(j)$ based on the TMTR vectors induced by the set of simple cycles $\Gamma$ in $H$. (Note that we can enumerate the states in $H$ so that the simple cycles in $\Gamma$ are ordered to begin with the smallest numbered state. This avoids listing a simple cycle more than once.)

Let $\eta$ be a cycle in $H$, which when decomposed into a collection of simple cycles, has $n_{t}$ copies of the $t$ th simple cycle $\gamma_{t}$, where $t=1, \ldots, k$, and $k$ is a positive integer $\leq|\Gamma|$. Let $i_{t}=i\left(\gamma_{t}\right), d_{t}=d\left(\gamma_{t}\right), q_{t}=q\left(\gamma_{t}\right)$, and $\nu_{t}=\nu\left(\gamma_{t}\right)$, denote the insertion rate, number of constrained positions, length of cycle label, and mean of the TMTR values of $\gamma_{t}$, respectively.

The length of the label of $\eta$ is $Q=\sum_{t=1}^{k} n_{t} q_{t}$, and the number of constrained positions in $\eta$ is $D=\sum_{t=1}^{k} n_{t} d_{t}$. The insertion rate of $\eta$ is $I=I(\eta)=1-D / Q$.

Let $W=W(\eta)$ denote the average of the TMTR values induced by $\eta$. Then

$$
W=\sum_{t=1}^{k} n_{t} d_{t} \nu_{t} / \sum_{t=1}^{k} n_{t} d_{t} \leq \max _{1 \leq t \leq k} \nu_{t} .
$$

Define $g_{t}=g\left(\gamma_{t}\right)=\left(1-i_{t}\right) \nu_{t}=\left(d_{t} \nu_{t}\right) / q_{t}$ for $t=1, \ldots, k$. Then $W$ becomes

$$
W=\frac{\sum_{t=1}^{k} n_{t} q_{t} g_{t}}{Q} \cdot \frac{1}{1-I}
$$

The factor $\sum_{t=1}^{k} n_{t} q_{t} g_{t} / Q$ is a convex combination of $g_{1}, \ldots, g_{k}$ and is upper-bounded (pointwise) by the convex hull $h$ of $\{(i(\gamma), g(\gamma)): \gamma \in \Gamma\}$, so

$$
W(\eta) \leq \frac{h(I(\eta))}{1-I(\eta)}
$$

Now for real $a>0$, define $z(a)$ to be $\log _{2}$ of the largest positive real root of

$$
x^{a+2}-2 x^{a+1}+1=0 .
$$

Since $z(a)$ is increasing with $a$ (see [4, Appendix]), we apply $z$ to (12) to obtain

$$
z(W(\eta)) \leq z\left(\frac{h(I(\eta))}{1-I(\eta)}\right) .
$$

Taking the supremum of both sides of inequality (13) over all cycles in $H$ that yield insertion rate $I(\eta)=x$, we obtain

$$
\sup _{\{\eta \in H: I(\eta)=x\}} z(W(\eta)) \leq z\left(\frac{h(x)}{1-x}\right) .
$$

Equation (14) leads to our formulation of an upper bound for the tradeoff function $f_{S}(x)$ for $S=\operatorname{MTR}(j)$.

Proposition 26: For insertion rate $\rho \in[0,1]$

$$
f_{S}(\rho) \leq(1-\rho) z\left(\left\lceil\frac{h(\rho)}{1-\rho}\right\rceil\right) .
$$

Proof: We make use of Theorem 44 of [11], which says that the tradeoff function $f$ at insertion rate $\rho$ can be achieved by a periodic configuration denoted by parameters $(U, N)$, where $U$ is the unconstrained set, and $N$ is the block length, and $|U| / N=$ $x$. This $(U, N)$ configuration corresponds to a cycle $\nu$ in $H$, and also induces a sequence of TMTR values which we can express as a vector $\boldsymbol{m}$.

Using Corollary 25, we have

$$
\begin{aligned}
f(\rho) & =f_{S_{U, N}}(\rho) \\
& =(1-\rho) \operatorname{cap}_{\overline{\text { TMTR }}}(\boldsymbol{m}) \\
& \leq(1-\rho) \sup _{\{\eta \in H: I(\eta)=\rho\}} z(\lceil W(\eta)\rceil) \\
& \leq(1-\rho) z\left(\left\lceil\frac{h(\rho)}{1-\rho}\right\rceil\right)
\end{aligned}
$$

where the third line is due to Theorem 16, and the fourth line comes from taking the ceiling on both sides in (13) before applying the function $z$ and taking the supremum on both sides over all cycles in $H$ with the same insertion rate.

We remark that the upper bound in Proposition 26 can be improved if part 2) of Conjecture is proven in the special case when $\max (\boldsymbol{m})=\min (\boldsymbol{m})+1$.

Example 27: For MTR (2), there are three states in $\mathrm{H}_{2}$, namely, $A B, B B$, and $A A$, and four possible edge labels $\{A B, A B B, A A B, A A B B\}$. The graph $\mathrm{H}_{2}$ is presented in Fig. 8, while the upper bound is plotted in Fig. 9 as a solid line in comparison with some upper and lower bounds from [11]. Namely, the dashed line corresponds to the bit-stuffing lower 


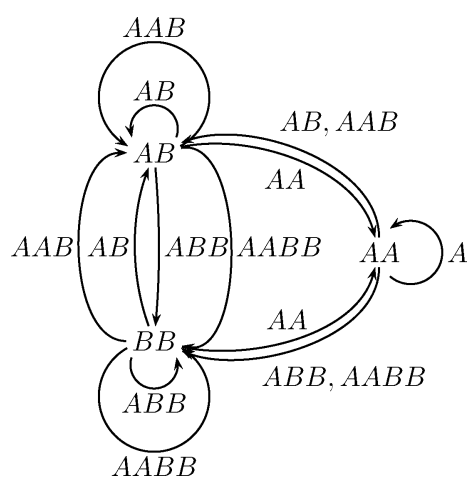

Fig. 8. Graph presentation of $H_{2}$ for MTR (2).

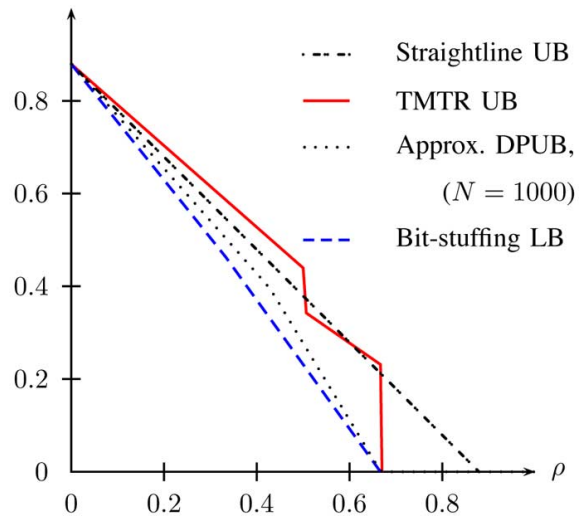

Fig. 9. Bounds on the tradeoff function of MTR (2).

bound (Bit-stuffing $L B$ ); the dotted line is the dynamic programming approximate upper bound with parameter $N=1000$ (Approx. DPUB); the dotted-dashed line corresponds to the only other known upper bound $f_{S}(\rho) \leq \operatorname{cap}_{S}-\rho$, which is a straight line and which we thus call the straightline $U B$. Note that the bit-stuffing lower bound (Bit-stuffing $L B$ ) for MTR (2) is the tradeoff function of MTR (2), as established by [4].

We present further plots of this new upper bound for MTR (3) and MTR (4) in Figs. 10 and 11, respectively. We remark that the tradeoff functions for MTR (3) and MTR (4) are not known. In fact, the tradeoff function for MTR $(j)$ with $j \geq 3$ is an open problem. From the plots, it can be seen that this TMTR upper bound improves on the straight-line upper bound over high insertion rates. This improvement is readily seen as $j$ increases. We can take the pointwise minimum of the two bounds to further obtain an improved upper bound.

One reason for the new TMTR upper bound underperforming in the low insertion rate region is because the average TMTR value $\bar{m}$ is generally not a good gauge of $\operatorname{cap}_{\text {TMTR }}(\boldsymbol{m})$. A TMTR vector can have a high $\bar{m}$ and yet a low capacity. For example, in the case of MTR (3), consider two configurations with the same insertion rate 0.4375: $\boldsymbol{m}_{1}=(0,0,0,0,1,2,3,3,2)$, which has average $\bar{m}_{1}=1.2222$ and capacity $\operatorname{cap}_{\mathrm{TMTR}}\left(\boldsymbol{m}_{1}\right)=0.5284$ versus $\boldsymbol{m}_{2}=(0,0,1,0,0,1,2,3,2)$, whose average $\bar{m}_{2}=1$ is strictly less than $\bar{m}_{1}$ but whose capacity $\operatorname{cap}_{\mathrm{TMTR}}\left(\boldsymbol{m}_{2}\right)=0.5342$ exceeds cap $\operatorname{mMTR}_{\text {TMT }}\left(\boldsymbol{m}_{1}\right)$.

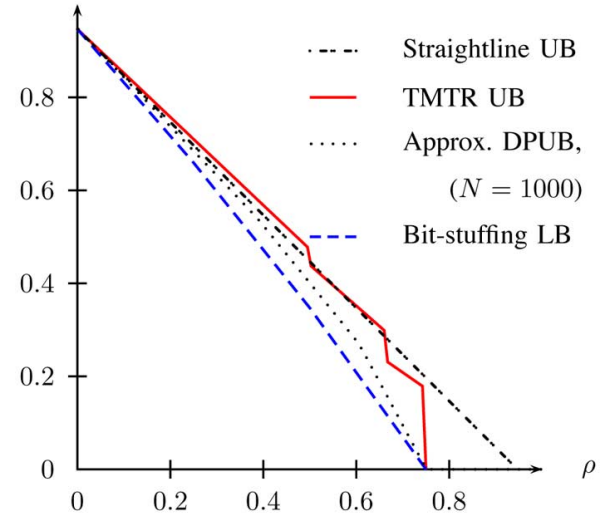

Fig. 10. Bounds on the tradeoff function of MTR (3).

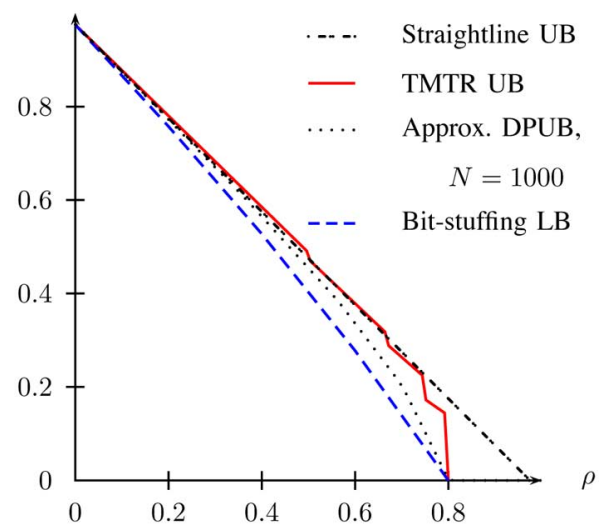

Fig. 11. Bounds on the tradeoff function of MTR (4).

\section{CONCLUSION}

In this paper, we have developed a general framework for analyzing and understanding time-varying maximum transition run constraints. We have computed bounds on the capacity of such constraints and used our results to obtain new bounds on the tradeoff function for constrained systems with unconstrained positions.

\section{APPENDIX}

Proof (Proof of Theorem 17): We first check the cases for which $\bar{m}<2$. For $\boldsymbol{m}=(1,2)$, the largest positive real root of its characteristic equation, as given by $(10)$, is $3^{1 / 2}<1.75<$ $\lambda(1.5)$. For $\boldsymbol{m}=(0,1,2)$, the largest positive real root of its characteristic equation, (9), is $2^{2 / 3}<1.618<\lambda(1)$. When $\boldsymbol{m}=(0,1,2,3)$, the largest positive real root of its characteristic equation is $2^{3 / 4}<1.754<\lambda(1.5)$. (We exclude the case $\boldsymbol{m}=(0,1)$ since the largest positive real root of its characteristic equation is $2^{1 / 2}>1.39>\lambda(0.5)$.)

Now assume $p \geq 2$ and $\bar{m}=M-(p-1) / 2 \geq 2$. Let $q=\lfloor(M+1) / p\rfloor$ and $r=(M+1) \bmod p$, so $M+1=q p+r$. The characteristic equation for TMTR $(\boldsymbol{m})$ for $\boldsymbol{m}$ satisfying the tightness condition with equality is given by (6), and can be rewritten as

$$
z^{q p}-\left(2^{p}-1\right) \frac{\left(z^{p}-z^{q p}\right)}{\left(1-z^{p}\right)}-\left(2^{p}-2^{p-1-r}\right)=0 .
$$


Multiplying by $\left(1-z^{p}\right)$ throughout and simplifying, we obtain

$$
\left(2^{p}-2^{p-1-r}\right)+\left(2^{p-1-r}-1\right) z^{p}+z^{M+1-r}\left(z^{p}-2^{p}\right)=0 .
$$

Let

$g(z)=\left(2^{p}-2^{p-1-r}\right)+\left(2^{p-1-r}-1\right) z^{p}+z^{M+1-r}\left(z^{p}-2^{p}\right)$.

Let $f(z)=z^{\bar{m}+2}-2 z^{\bar{m}+1}+1$, where $\bar{m}=M-$ $(p-1) / 2$ as above, so $f(z)=z^{M+1-r} z^{r-(p-1) / 2}(z-2)+1$. Note that if $\bar{m} \in \mathbb{Z}$, then $f(z)=0$ is the characteristic equation of $\operatorname{MTR}(\bar{m})$. The equation $f(z)=0$ has a unique solution $z=z(\bar{m})$ in $(1,2)$ and the solution is increasing as a function of $\bar{m}$ (see [4, Proof of Lemma 2]).

Let $z_{0}=1.83$, chosen so that $\operatorname{cap}(\operatorname{MTR}(2))>z_{0}$. It follows that the root of $f$ in $(1,2)$ is greater than $z_{0}$ since $\bar{m} \geq 2$. It suffices to show that for any $\alpha \in\left[z_{0}, 2\right), g(\alpha)=0$ implies $f(\alpha)<0$, because $f$ is continuous and $f(2)=1>0$.

Assume that $g(\alpha)=0$, where $\alpha$ is in $\left[z_{0}, 2\right)$. Then

$$
\alpha^{M+1-r}=-\frac{\left(2^{p}-2^{p-1-r}\right)+\left(2^{p-1-r}-1\right) \alpha^{p}}{\left(\alpha^{p}-2^{p}\right)} .
$$

Substituting the above equation into $f$, we obtain

$$
\begin{aligned}
& f(\alpha) \\
& =-\left(\frac{\left(2^{p}-2^{p-1-r}\right)+\left(2^{p-1-r}-1\right) \alpha^{p}}{\left(\alpha^{p}-2^{p}\right)}\right) \alpha^{r-\frac{p-1}{2}}(\alpha-2) \\
& \quad+1 .
\end{aligned}
$$

We will show that $f(\alpha)<0$. This is equivalent to showing

$$
\begin{aligned}
& \alpha^{p}-2^{p}-\left(\left(2^{p}-2^{p-1-r}\right)\right. \\
& \left.+\left(2^{p-1-r}-1\right) \alpha^{p}\right)(\alpha-2) \alpha^{r-\frac{p-1}{2}}>0 .
\end{aligned}
$$

For $p=2$, consider the two cases $r=0$ and $r=1$. When $r=1$, inequality (15) reduces to $(\alpha-2)\left((\alpha+2)-3 \alpha^{1 / 2}\right)>0$, which is equivalent to $(\alpha+2)-3 \alpha^{1 / 2}<0$, since $\alpha<2$. The latter is equivalent to $(\alpha-4)(\alpha-1)=\alpha^{2}-5 \alpha+4<0$, which is true for $\alpha \in(1,4)$.

When $r=0$, inequality (15) reduces to

$$
(\alpha-2)\left((\alpha+2)-\left(2+\alpha^{2}\right) \alpha^{-1 / 2}\right)>0
$$

which is equivalent to $(\alpha+2)-\left(2+\alpha^{2}\right) \alpha^{-1 / 2}<0$, which is in turn equivalent to $\alpha^{4}-\alpha^{3}-4 \alpha+4>0$. The latter is true for $\alpha \in\left(z_{0}, 2\right)$ since its largest real root is $1.5874<z_{0}$.

For $p \geq 3$, first note that

$$
\begin{aligned}
\alpha^{p}-2^{p}=(\alpha-2)\left(\alpha^{p-1}+\alpha^{p-2} 2+\alpha^{p-3} 2^{2}\right. & \left.+\cdots+2^{p-1}\right) \\
& \geq(\alpha-2) p 2^{p-1}
\end{aligned}
$$

since $\alpha-2<0$. Thus, the left-hand side of inequality (15) is at least

$$
\begin{aligned}
(\alpha-2) p 2^{p-1}-\left(2^{p}-2^{p-1-r}\right)(\alpha-2) \alpha^{r-\frac{p-1}{2}} & \\
& +\left(2^{p-1-r}-1\right) \alpha^{p}(\alpha-2) \alpha^{r-\frac{p-1}{2}} \\
= & (\alpha-2) 2^{p-1-r} . \\
& \left(p 2^{r}-\left(\left(2^{r+1}-1\right)+\left(1-\frac{1}{2^{p-1-r}}\right) \alpha^{p}\right) \alpha^{r-\frac{p-1}{2}}\right),
\end{aligned}
$$

so it suffices to show

$$
\left(2^{r+1}-1\right) \alpha^{r-\frac{p-1}{2}}+\left(1-\frac{1}{2^{p-1-r}}\right) \alpha^{r+\frac{p+1}{2}}>p 2^{r} .
$$

For $p<14$, this inequality is easily checked by explicit calculation using

$$
\begin{aligned}
\left(2^{r+1}-1\right) \min \left(1.83^{r-\frac{p-1}{2}}, 2^{r-\frac{p-1}{2}}\right) & \\
& +\left(1-\frac{1}{2^{p-1-r}}\right)(1.83)^{r+\frac{p+1}{2}}
\end{aligned}
$$

as a lower bound to the left-hand side of inequality (16) by letting $p$ range from 3 to 8 and $r$ range from 0 to $p-1$.

Assume $p \geq 14$, and consider the two cases $r=p-1$ and $r<p-1$ separately. For $r=p-1$, inequality (16) reduces to $\left(2^{p}-1\right) \alpha^{\frac{p-1}{2}}>p 2^{p-1}$, which is true since $2^{p}-1>2^{p-1}$ and

$$
\alpha^{(p-1) / 2}>(\sqrt{1.83})^{p-1}>1.352^{p-1}>p, \quad \text { for } p \geq 8 .
$$

For $r<p-1$, we will show $\left(1-\frac{1}{2^{p-1-r}}\right) \alpha^{r+\frac{p+1}{2}}>p 2^{r}$, which implies inequality (16) since the term $\left(2^{r+1}-1\right) \alpha^{r-\frac{p-1}{2}}$ is also positive.

Note that $1-1 /\left(2^{p-1-r}\right) \geq 1 / 2$, so it suffices to show

$$
\left(\frac{\alpha}{2}\right)^{r} \alpha^{\frac{p+1}{2}}>2 p \text {. }
$$

We can then bound the left-hand side of inequality (17) as follows:

$$
\begin{aligned}
\left(\frac{\alpha}{2}\right)^{r} \alpha^{\frac{p+1}{2}} & \geq\left(\frac{\alpha}{2}\right)^{p-2} \alpha^{\frac{p+1}{2}} \\
& =2^{2-p} \alpha^{(3 p-3) / 2} \\
& \geq 2^{2-p} z_{0}^{(3 p-3) / 2}
\end{aligned}
$$

Note that

$$
2 z_{0}^{(3 p-3) / 2}=2\left(z_{0}^{3 / 2}\right)^{p} z_{0}{ }^{-3 / 2} \geq\left(2.475^{p}\right)(0.8)>p 2^{p}
$$

for $p \geq 14$ since $(2.475 / 2)^{p}=1.2375^{p}>1.25 p$ for $p \geq 14$. Thus, $2^{2-p} z_{0}{ }^{(3 p-3) / 2}>2 p$, which proves inequality (17).

To prove part 1) of Proposition 22, we require the following lemma.

Lemma 28: Let $\lambda_{0}$ be the largest positive real root of the characteristic equation of $\operatorname{TMTR}(t, t, t+1, t+1)$. Then $\lambda_{0}$ is given by the largest positive real root of the following equations (one per congruence class of $t$ ):

$$
3+12 z^{4+8 s}-16 z^{4+12 s}+z^{8+12 s}=0
$$

for $t=4 s$;

$$
2+7 z^{4+4 s}+6 z^{8+8 s}-16 z^{8+12 s}+z^{12+12 s}=0
$$

for $t=4 s+1$;

$$
-4+z^{4}+16 z^{4+4 s}+2 z^{8+4 s}-16 z^{8+8 s}+z^{12+8 s}=0
$$


for $t=4 s+2$;

$$
1-10 z^{4+4 s}+24 z^{8+8 s}-16 z^{12+12 s}+z^{16+12 s}=0
$$

for $t=4 s+3$, where $s$ is a nonnegative integer. follows:

Proof: First define the polynomials $p(z), q(z)$, and $r(z)$ as

$$
\begin{aligned}
& p(z)=\sum_{i=0}^{s} z^{-4 i}=\frac{z^{-(4 s+4)}-1}{z^{-4}-1} \\
& q(z)=\sum_{i=0}^{s-1} z^{-4 i}=\frac{z^{-4 s}-1}{z^{-4}-1} \\
& r(z)=\sum_{i=0}^{s+1} z^{-4 i}=\frac{z^{-(4 s+8)}-1}{z^{-4}-1} .
\end{aligned}
$$

Construct the VLG of the standard trellis presentation of TMTR $(t, t, t+1, t+1)$ by choosing the four trellis states $\{(0,0),(1,0),(2,0),(3,0)\}$ as the Rome set. By counting the paths and path lengths of first return to the Rome set, we form the matrix (one for each congruence class of $t$ ) whose characteristic equation is given by (4). Let $I$ denote the $4 \times 4$ identity matrix.

For $t=4 s+i$, where $i=0,1,2,3$, the determinantal equations are given by $\left|V_{i}-I\right|=0$, respectively, with the matrices $V_{i}(i=0,1,2,3)$ listed as follows:

$$
\begin{aligned}
& V_{0}=\left[\begin{array}{llll}
z^{-4} q(z) & z^{-1} p(z) & z^{-2} q(z) & z^{-3} q(z) \\
z^{-3} q(z) & z^{-4} q(z) & z^{-1} p(z) & z^{-2} p(z) \\
z^{-2} p(z) & z^{-3} q(z) & z^{-4} q(z) & z^{-1} p(z) \\
z^{-1} p(z) & z^{-2} q(z) & z^{-3} q(z) & z^{-4} q(z)
\end{array}\right] \\
& V_{1}=\left[\begin{array}{llll}
z^{-4} q(z) & z^{-1} p(z) & z^{-2} p(z) & z^{-3} p(z) \\
z^{-3} p(z) & z^{-4} q(z) & z^{-1} p(z) & z^{-2} p(z) \\
z^{-2} p(z) & z^{-3} q(z) & z^{-4} q(z) & z^{-1} p(z) \\
z^{-1} p(z) & z^{-2} p(z) & z^{-3} q(z) & z^{-4} q(z)
\end{array}\right] \\
& V_{2}=\left[\begin{array}{llll}
z^{-4} p(z) & z^{-1} p(z) & z^{-2} p(z) & z^{-3} p(z) \\
z^{-3} p(z) & z^{-4} q(z) & z^{-1} p(z) & z^{-2} p(z) \\
z^{-2} p(z) & z^{-3} p(z) & z^{-4} q(z) & z^{-1} p(z) \\
z^{-1} p(z) & z^{-2} p(z) & z^{-3} p(z) & z^{-4} p(z)
\end{array}\right] \\
& V_{3}=\left[\begin{array}{llll}
z^{-4} p(z) & z^{-1} p(z) & z^{-2} p(z) & z^{-3} p(z) \\
z^{-3} p(z) & z^{-4} p(z) & z^{-1} p(z) & z^{-2} p(z) \\
z^{-2} p(z) & z^{-3} p(z) & z^{-4} p(z) & z^{-1} r(z) \\
z^{-1} r(z) & z^{-2} p(z) & z^{-3} p(z) & z^{-4} p(z)
\end{array}\right] .
\end{aligned}
$$

It is easy to check that the determinantal equations expand to (18), (19), (20), and (21), respectively, for $t=4 s, 4 s+1,4 s+$ $2,4 s+3$.

Proof (Proof of Part 1) of Proposition 22):

1a) Let $t=4 s+2$. We can factorize (20) from Lemma 28 as follows: implying

$$
\left(z^{4 s+6}-4 z^{4 s+4}+z^{2}+2\right)\left(z^{4 s+6}+4 z^{4 s+4}+z^{2}-2\right)=0
$$

which in turn implies

$$
\begin{aligned}
\left(z^{4 s+4}-3 \sum_{k=1}^{2 s+1} z^{4 s+4-2 k}-2\right) & \\
\left(z^{4 s+4}-3 \sum_{k=1}^{2 s+1}(-1)^{k} z^{4 s+4-2 k}-2\right) & =0 .
\end{aligned}
$$

Let

$$
f(z)=z^{t+2}-3 \sum_{k=1}^{\frac{t}{2}} z^{t+2-2 k}-2
$$

and

$$
g(z)=z^{t+2}-3 \sum_{k=1}^{\frac{t}{2}}(-1)^{k} z^{t+2-2 k}-2 .
$$

Note that $f(z)=g(i z)$ and $g(z)=f(-i z)$, so the moduli of the roots of $f(z)$ are the same as the moduli of the roots of $g(z)$. But $f(z)$ is the characteristic polynomial of TMTR $(t, t+1)$ by (7). Thus, $\operatorname{cap}_{\overline{\mathrm{TMTR}}}(t, t+1)=\operatorname{cap}_{\overline{\mathrm{TMTR}}}(t, t, t+1, t+1)$.

1b) From (7), the characteristic polynomial of $\overline{\mathrm{TMTR}}(t, t+1)$ for $t=4 s$ is given by $q(z)=z^{4 s+2}-3 \sum_{k=1}^{2 s} z^{2 k}-2$ which simplifies to $z^{4 s+2}-3 \frac{\left(z^{4 s+2}-z^{2}\right)}{z^{2}-1}-2$. Define the polynomial $g(z)$ as

$$
g(z)=\left(z^{2}-1\right) q(z)=2+z^{2}-4 z^{4 s+2}+z^{4 s+4} .
$$

Let $f(z)$ denote the function

$$
f(z)=3+12 z^{4+8 s}-16 z^{4+12 s}+z^{8+12 s} .
$$

From Lemma 28 (18), the largest positive real root of $f(z)=0$ equals the largest positive real root of the characteristic equation of $\overline{\operatorname{TMTR}}(t, t, t+1, t+1)$ for $t=4 s$. Note also that $g(z)=0$ has the same largest root as $q(z)=0$. Observe that $g(2)=6>$ 0 , and $g(\sqrt{2})=4\left(1-2^{2 s}\right) \leq 0$ (with strict inequality for all $s \geq 1)$. The derivative $g^{\prime}(z)$ is $2 z+4 z^{4 s+1}((z-2)(z+2) s+$ $\left.\left(z^{2}-2\right)\right)>0$ for $z \geq 2$, so $g$ is increasing on $(2, \infty)$. Thus, the largest root of $g$ is contained in $[\sqrt{2}, 2)$.

Perform a change of variables on $f$ and $g$ by letting $y=z^{2}$. We obtain

$$
\begin{aligned}
f(y) & =3+12 y^{2+4 s}-16 y^{2+6 s}+y^{4+6 s} \\
& =3+12 y^{2+4 s}+\left(y^{2 s+1}(y-4)\right)\left(y^{4 s+1}(y+4)\right)
\end{aligned}
$$

and $g(y)=2+y+y^{2 s+1}(y-4)$. So we can restrict attention to finding a root in $[2,4)$.

Setting $f(y)=0$, the solution $y=y_{0}$ satisfies

$$
y^{2 s+1}(y-4)=-\frac{\left(3+12 y^{2+4 s}\right)}{y^{4 s+1}(y+4)} .
$$


Then, substituting the above into $g(y)$, we obtain

$$
g\left(y_{0}\right)=2+y_{0}-\frac{\left(3+12 y_{0}^{2+4 s}\right)}{y_{0}^{4 s+1}\left(y_{0}+4\right)} .
$$

We claim that $g(y)<0$ for $y$ in the open interval $(2,4)$. This is because the function $y^{4 s+1}(y-4)(y-2)-3<0$ for $y \in(2,4)$. Rewriting $y^{4 s+1}(y-4)(y-2)-3$ as $y^{4 s+3}-$ $6 y^{4 s+2}+8 y^{4 s+1}-3$, yields $\left(y^{2}+6 y+8\right) y^{4 s+1}<3+12 y^{2+4 s}$, which implies that

$$
2+y-\frac{\left(3+12 y^{2+4 s}\right)}{y^{4 s+1}(y+4)}<0
$$

which is equivalent to $g(y)<0$.

Since $y_{0}$ falls into the range $(2,4)$, this implies that $g\left(y_{0}\right)<0$ for $f\left(y_{0}\right)=0$. Thus, the largest root of $g$ is strictly to the right of the largest root of $f$, hence

$\operatorname{cap}_{\overline{\mathrm{TMTR}}}(t, t+1)>\operatorname{cap}_{\overline{\mathrm{TMTR}}}(t, t, t+1, t+1)$, for $t=4 s$.

1c) Let $t=4 s+1$. Let $f$ denote the function $f(z)=z^{12 s}\left(z^{12}-16 z^{8}\right)+6 z^{8 s+8}+7 z^{4 s+4}+2$. From Lemma 28 (19), the largest positive real root of $f(z)=0$ equals the largest positive real root of the characteristic equation of $\overline{\mathrm{TMTR}}(t, t, t+1, t+1)$ for $t=4 s+1$. Observe that $f(2)>0$ while $f(1.7)<0$ (It is easily checked that for $s=0$, the largest positive real root of $\overline{\operatorname{TMTR}}(1,1,2,2)$ is 1.7424$)$. We compute the derivative

$$
\begin{aligned}
f^{\prime}(z)=4 z^{4 s+3}(7 s+ & 7+(12 s+12) z^{4 s+4} \\
& \left.+z^{8 s+4}\left((3 s+3)\left(z^{4}-16\right)+16\right)\right)>0
\end{aligned}
$$

for $z \geq 2$, so $f$ is increasing on $[2, \infty)$. Thus, the largest positive real root of $f$ lies in the open interval $(1.7,2)$.

From (7), the characteristic polynomial of $\overline{\operatorname{TMTR}}(t, t+1)$ for $t=4 s+1$ is given by

$$
q(z)=z\left(z^{4 s+2}-3 \frac{z^{4 s+2}-1}{z^{2}-1}\right) .
$$

For $q(z)=0, z=0$ or $z^{4 s+2}-3 \frac{z^{4 s+2}-1}{z^{2}-1}=0$, of which the latter reduces to

$$
z^{4 s}=-\frac{3}{z^{4}-4 z^{2}} .
$$

Thus, substituting (22) into $f(z)$, we obtain

$$
\begin{aligned}
f(z)=(- & \left.\frac{3}{z^{4}-4 z^{2}}\right)^{3}\left(z^{12}-16 z^{8}\right) \\
& +6 z^{8}\left(-\frac{3}{z^{4}-4 z^{2}}\right)^{2}+7 z^{4}\left(-\frac{3}{z^{4}-4 z^{2}}\right)+2 .
\end{aligned}
$$

We claim that $f(z)<0$ for $z \in(1.7,2)$. Note that showing $f(z)<0$ is equivalent to showing $\left(z^{4}-4 z^{2}\right) f(z)>0$ for $z \in$ $(1.7,2)$. But it is easily checked that $\left(z^{4}-4 z^{2}\right) f(z)$ simplifies to $8 z^{6}\left(z^{2}-4\right)^{2}\left(z^{2}-1\right)>0$, hence proving the claim. Thus, the largest root of $f$ is strictly to the right of that of $q$.
Let $t=4 s+3$. Let $f$ denote the function

$$
f(z)=z^{12 s+12}\left(z^{4}-16\right)+24 z^{8 s+8}-10 z^{4 s+4}+1 .
$$

From Lemma 28 (21), the largest positive real root of $f(z)=0$ equals the largest positive real root of the characteristic equation of $\overline{\text { TMTR }}(t, t, t+1, t+1)$ for $t=4 s+3$. Also observe that $f(2)>0$, while $f(1.9)<0$ (It is easily checked that for $s=0$, the largest positive real root of $\overline{\operatorname{TMTR}}(1,1,2,2)$ is 1.9473$)$. We compute the derivative

$$
\begin{aligned}
f^{\prime}(z)=4 z^{4 s+3}(2(s+1) & \left(24 z^{4 s+4}-5\right) \\
+ & \left.z^{8 s+8}\left(z^{4}(3 s+4)-48(s+1)\right)\right)>0
\end{aligned}
$$

for $z \geq 2$, so $f$ is increasing on $[2, \infty]$. Thus, the largest positive real root of $f$ lies in the open interval $(1.9,2)$.

From (7), the characteristic polynomial of $\overline{\mathrm{TMTR}}(t, t+1)$ for $t=4 s+3$ is given by

$q(z)=z\left(z^{4 s+4}-3 \sum_{k=0}^{2 s+1} z^{2 k}\right)=z\left(z^{4 s+4}-3 \frac{z^{4 s+4}-1}{z^{2}-1}\right)$.

For $q(z)=0, z=0$ or $z^{4 s+4}-3 \frac{z^{4 s+4}-1}{z^{2}-1}=0$, of which the latter reduces to

$$
z^{4 s+4}=-\frac{3}{z^{2}-4}
$$

Thus, substituting (23) into $f(z)$, we obtain

$$
\begin{aligned}
f(z)=\left(-\frac{3}{z^{2}-4}\right)^{3}\left(z^{4}-16\right) & \\
& +24\left(-\frac{3}{z^{2}-4}\right)^{2}-10\left(-\frac{3}{z^{2}-4}\right)+1 .
\end{aligned}
$$

We claim that $f(z)<0$ for $z \in(1.9,2)$. Note that showing $f(z)<0$ is equivalent to showing $\left(z^{2}-4\right) f(z)>0$ for $z \in$ $(1.9,2)$. It turns out that

$$
\begin{aligned}
\left(z^{2}-4\right) f(z) & =-27\left(z^{2}-4\right)\left(z^{2}+4\right)+246+\left(z^{2}-4\right), \\
& =\left(4-z^{2}\right)\left(27 z^{2}+107\right)+246>0
\end{aligned}
$$

for $z<2$, thus proving the claim.

Thus, the largest root of $f$ is strictly to the right of the largest root of $q$. Hence, $C(t, t+1)<C(t, t, t+1, t+1)$ for $t=4 s+1$ or $t=4 s+3$.

Parts 2) through 6) of Proposition 22 can be proved using the same techniques and are thus omitted here.

\section{ACKNOWLEDGMENT}

T. L. Poo wishes to thank J. K. Blitzstein for many helpful discussions.

\section{REFERENCES}

[1] R. L. Adler, D. Coppersmith, and M. Hassner, "Algorithms for sliding block codes-An application of symbolic dynamics to information theory," IEEE Trans. Inf. Theory, vol. IT-29, no. 1, pp. 5-22, Jan. 1983. 
[2] R. L. Adler, J. Friedman, B. Kitchens, and B. H. Marcus, "State splitting for variable-length graphs," IEEE Trans. Inf. Theory, vol. IT-32, no. 1, pp. 108-113, Jan. 1986.

[3] W. Bliss, "An 8/9 rate time-varying trellis code for high density magnetic recording," IEEE Trans. Magn., vol. 33, no. 5, pp. 2746-2748, Sep. 1997.

[4] J. C. de Souza, B. H. Marcus, R. New, and B. A. Wilson, "Constrained systems with unconstrained positions," IEEE Trans. Inf. Theory, vol. 48, no. 4, pp. 866-879, Apr. 2002.

[5] K. K. Fitzpatrick and C. S. Modlin, "Time-varying mtr codes for high density magnetic recording," in Proc. IEEE Globecom Telecomm. Conf., Phoenix, AZ, Nov. 1997, pp. 1250-1253.

[6] R. Karabed, P. H. Siegel, and E. Soljanin, "Constrained coding for binary channels with high intersymbol interference," IEEE Trans. Inf. Theory, vol. 45, no. 6, pp. 1777-1797, Sep. 1999.

[7] B. H. Marcus, R. M. Roth, and P. H. Siegel, Handbook of Coding Theory. Amsterdam, The Netherlands: Elsevier, 1998, ch. 20.

[8] B. E. Moision, P. H. Siegel, and E. Soljanin, "Distance-enhancing codes for digital recording," IEEE Trans. Magn., vol. 34, no. 1, pp. 69-74, Jan. 1998
[9] J. Moon and B. Brickner, "Maximum transition run codes for data storage systems," IEEE Trans. Magn., vol. 32, no. 5, pp. 3992-3994, Sep. 1996.

[10] T. L. Poo, "Optimal code rates for constrained systems with unconstrained positions: An approach to combining error correction codes with modulation codes for digital storage systems," Ph.D. dissertation, Stanford Univ., Stanford, CA, 2005.

[11] T. L. Poo, P. Chaichanavong, and B. H. Marcus, "Tradeoff functions for constrained systems with unconstrained positions," IEEE Trans. Inf. Theory, vol. 52, no. 4, pp. 1425-1449, Apr. 2006.

[12] C. E. Shannon, "A mathematical theory of communications," Bell Syst. Tech. J., vol. 27, pp. 379-423, 1948.

[13] A. J. van Wijngaarden and K. A. S. Immink, "Maximum runlength-limited codes with error control capabilities," IEEE J. Sel. Areas Commun., vol. 19, no. 4, pp. 602-611, Apr. 2001. 